CERN-PPE/95-10

CERN-SL/95-02

20 January 1995

\title{
The Energy Calibration of LEP in the 1993 Scan
}

\author{
The working group on LEP energy
}

R. Assmann ${ }^{1, *)}$, A. Beuret ${ }^{2)}$, A. Blonde ${ }^{3)}$, J. Billan ${ }^{2)}$, R. Billen ${ }^{2)}$, G.J. Bobbink ${ }^{4)}$, F. Bordry ${ }^{2)}$, H. Burkhardt ${ }^{2)}$, B. Dehning ${ }^{2)}$, A. Drees ${ }^{5)}$, J. Gascon ${ }^{6)}$, P. Grosse-Wiesmann ${ }^{2)}$, H. Grote ${ }^{2)}$, C.M. Hawkes ${ }^{2)}$, K. Henrichsen ${ }^{2)}$, A. Hofmann ${ }^{2)}$, R. Jacobsen ${ }^{2+}+$, M. Jonker ${ }^{2)}$, M. Koratzinos ${ }^{7)}$, J.P. Koutchouk ${ }^{2)}$, J. Miles ${ }^{2)}$, S. Myers ${ }^{2)}$, E. Peschardt ${ }^{2)}$, M. Placidi ${ }^{2)}$, D. Plane ${ }^{2)}$, G. Quast ${ }^{8)}$, P. Renton ${ }^{9)}$, L. Rolandi ${ }^{2)}$, R. Schmidt ${ }^{2)}$, D. Strom ${ }^{10)}$, R. Veenhof ${ }^{2, x)}$, M. Vadon ${ }^{2)}$, H. Wachsmuth ${ }^{2)}$, J. Wenninger ${ }^{2)}$, T. Wyatt ${ }^{11)}$

\begin{abstract}
This report summarizes the procedure for providing the absolute energy calibration of the LEP beams during the energy scan in 1993. The average beam energy around the LEP ring was measured in 25 calibrations with the resonant depolarization technique. The time variation of this average beam energy is well described by a model of the accelerator based on monitored quantities. The absolute calibration of the centre of mass energies of the off-peak points is determined with a precision of 2 parts in $10^{5}$ resulting in a systematic error on the Z-mass of about $1.4 \mathrm{MeV}$ and on the Z-width of about $1.5 \mathrm{MeV}$.
\end{abstract}

\section{(Submitted to Zeitschrift für Physik C)}

\footnotetext{
1) Max-Plank-Institut für Pysik, Werner-Heisenberg-Institut, D-80805 München, Germany

2) European Laboratory for Particle Physics (CERN), CH-1211 Geneva 23, Switzerland

3) Laboratoire de Physique Nucléaire et des Hautes Energies, Ecole Polytechnique, IN ${ }^{2} \mathrm{P}^{3} \mathrm{CRNS}_{\mathrm{C}}$ F91128 Palaiseau Cedex, France

4) National Institute for High Energy Physics, NIKHEF, NL-1009 DB Amsterdam, The Netherlands

5) Fachbereich Physik, Univeristy of Wuppertal, Postfach 100 127, D-42097, Wuppertal, Germany

6) Laboratoire de Physique Nucléaire, Université de Montréal, Montréal, Quebec, H3C3J7, Canada

7) Physics Department, University of Oslo, Blindern, N-1000 Oslo 3, Norway

8) Institut für Physik, Universität Mainz, D-55099 Mainz, Germany

9) Department of Physics, University of Oxford, Keble Road, Oxford OX1 3RH, UK

10) University of Oregon, Dept. of Physics, Eugene, Oregon 97403, USA

11) Departement of Physics, Schuster Laboratory, The University, Manchester, M139PL, UK

*) Present address: Stanford Linear Accelerator Center (SLAC), Stanford Univ., P.O. Box 4349, Stanford, CA 94309, USA

+) Present address: Dept. of Physics, Univ. of California, Berkeley CA 94720, USA

x) Present address: Laboratório de Instrumentação e Física Experimental de Partículas, Av. E. Garcia 14-1, P-1000 Lisboa, Portugal
} 
During the 1993 run of the LEP electron-positron collider at CERN an energy scan was performed around the $Z$ resonance. This followed an initial period during which the method of beam energy calibration using resonant depolarization [1] was commissioned [2].

This paper describes how the centre of mass energies of the colliding beams at the four interaction points were measured during this scan, allowing a more precise determination of the $\mathrm{Z}$ mass and width. This was possible because a large fraction of the fills were calibrated at the end of the coast and, in addition, many parameters which could in principle affect the energy of the beams were continuously monitored and logged [3]. The precision on the $\mathrm{Z}$ mass from the scan in 1991 was systematically limited by centre of mass energy calibration [4] and is significantly improved with the new calibration. The calibration of two energy points on the line shape and the increase in statistics also give a significant reduction of the error on the $Z$ width.

Data were taken at three scan points with different centre of mass energies (named peak -2 , peak and peak +2 ) placed almost symmetrically around the $Z$ peak and each separated by roughly $1790 \mathrm{MeV}$ from it. The cross sections at the two off-peak points were typically measured in adjacent fills interspersed with measurements at the peak. The integrated luminosities delivered to each of the four experiments at the three points were approximately $10 \mathrm{pb}^{-1}, 20 \mathrm{pb}^{-1}$ and $10 \mathrm{pb}^{-1}$. The statistical errors on the $\mathrm{Z}$ mass and width from this data sample are $2 \mathrm{MeV}$ and $3 \mathrm{MeV}$ respectively, with negligible experimental systematics [5], while the systematic errors from energy calibration depend approximately on the errors on the sum and on the difference of centre of mass energies at the two off-peak points.

$$
\begin{gathered}
\Delta \mathrm{M}_{\mathrm{Z}} \approx 0.5 \Delta\left(\mathrm{E}_{+2}+\mathrm{E}_{-2}\right) \\
\Delta \Gamma_{\mathrm{Z}} \approx \frac{\Gamma_{\mathrm{Z}}}{\left(\mathrm{E}_{+2}-\mathrm{E}_{-2}\right)} \Delta\left(\mathrm{E}_{+2}-\mathrm{E}_{-2}\right)=0.71 \Delta\left(\mathrm{E}_{+2}-\mathrm{E}_{-2}\right)
\end{gathered}
$$

where $\mathrm{E}_{-2}$ and $\mathrm{E}_{+2}$ are the luminosity-weighted centre of mass energies at the two off-peak points. These formulae hold under the assumption that the "peak" luminosity is collected at the point of maximum cross section of the $Z$ resonance. The values of $E_{-2}$ and $E_{+2}$ have to be known with an error of $0.002 \%$ or better to match the statistical precision of the measurements.

The off-peak luminosity was collected in 38 fills at peak-2 and 31 fills at peak +2 of which 13 and 11 respectively were calibrated at end of the coast, using the resonant depolarization method. This method, which is discussed in section 1, gives the average energy of the circulating electron beam at the time of the calibration with an error below $1 \mathrm{MeV}$. The energy of the positron beam is assumed to be equal to the energy of the electron beam with a constant offset. The difference between the energies of the two beams has been measured in dedicated experiments discussed in section 2.

The difference between the average beam energy and the beam energy at the interaction points depends on the geometrical alignment and on the status of the RF accelerating system. The RF units are placed symmetrically to the left and right of the L3 and OPAL experiments and are used during the coast to compensate for the energy lost due to synchrotron radiation. Their alignment is well known and the operating status of the RF units was monitored and logged during the scan resulting in an almost negligible systematic error, as discussed in section 3 .

The combination of the calibration using resonant depolarization and the interaction point dependent corrections gives the centre of mass energies of the colliding beams 
at the time of calibration with a systematic error at the $1 \mathrm{MeV}$ level. A number of effects discussed below cause the energy of the circulating beams to vary with time. However, since the calibrations at each scan point sample the integrated luminosities in an almost unbiased way with respect to these effects, their mean values are almost unbiased estimators of the two energies, $\mathrm{E}_{-2}$ and $\mathrm{E}_{+2}$. Their statistical errors are estimated from the RMS of the distributions and the number of calibrations. Since considerable variations in the LEP energy were observed during the course of the scan, the RMS variations of the centre of mass energies are as large as $15 \mathrm{MeV}$, resulting in statistical errors of about $4 \mathrm{MeV}$ at each energy point.

This error is large compared to the required precision, but can be reduced if sources of variation are identified and related to monitored quantities. The time variation of the average beam energy is then modelled as a function of these quantities and this function is used to fit the energies measured with the resonant depolarization method. Only one parameter is left free at each scan point to define the overall energy scale. The statistical errors on $\mathrm{E}_{-2}$ and $\mathrm{E}_{+2}$ are reduced to the extent that the model is a good description of the observed time variation of the beam energy and the extra uncertainty introduced by the quantities used to predict the energy is small.

This model, which is discussed in section 4, describes the variations with time of the integrated vertical magnetic field seen by the beam particles along their trajectories. There are two main effects that can change the integrated bending field: a variation of the average relative position of the beam with respect to the centre of the quadrupole magnets and a change of the magnetic field of the dipole magnets. The first effect can be caused by a change of the dimension of the ring with respect to the length of the closed orbit which is determined by the accelerating RF frequency. These changes are induced on the time scale of a day by the earth tides [6] or by other causes on longer time scales. This effect can be monitored by measuring the variation of the position of the beams with respect to the centre of the quadrupoles using the beam position pickups. The energy variations induced by these effects are large: about $1 \mathrm{MeV}$ for an average displacement of $13 \mu \mathrm{m}$, corresponding to centre of mass energy variations of $20 \mathrm{MeV}$ for large tides. The changes of the integrated magnetic field of the dipoles are monitored by measuring the currents, the magnetic field in an instrumented reference magnet and the temperature of the magnets in the ring. They also produce centre of mass energy changes in the range of $10 \mathrm{MeV}$ full scale.

The RMS variation of the deviations between the fitted model and the calibration data gives a measurement of the unmodelled effects, including any possible nonreproducibility of the LEP settings. It is $5.4 \pm 0.8 \mathrm{MeV}$, a factor of about three smaller than the RMS of the calibration data.

The systematic errors on the luminosity-weighted energies and their correlations are discussed in section 5. In addition to the uncertainty arising from the deviations between the fitted model and the calibrations, the main sources of systematic error are uncertainties in the RF and temperature corrections, systematic errors in the measurement of the average energy using resonant depolarization, uncertainty in the energy difference between the two beams and possible systematic changes in the integrated dipole field during the time each fill is kept inside LEP.

Section 6 describes various tests that have been performed to check the calibration procedure and the measurements on which it is based. Section 7 describes the calibration of the peak data collected in 1993 before the scan. Section 8 is a review of the previous LEP calibrations $[7,8]$ and a comparison with the present one. 


\section{Energy calibration by resonant depolarization}

In an ideal $\mathrm{e}^{+} \mathrm{e}^{-}$storage ring the beams naturally polarize along the direction of the bending field due to the emission of synchrotron radiation [9]. The polarization vector is defined as the ensemble average of the spin vectors of all the electrons in the bunch. The spin vector of each electron precesses on average $a_{e} \gamma$ times during one turn around the ring, where $a_{e}$ is the electron magnetic moment anomaly and $\gamma$ is the average Lorentz factor of this electron. The spin tune is defined as $a_{e} \gamma$ and the time-averaged spin tune, $\nu_{0}$, of each electron is proportional to the average beam energy, $E$ :

$$
\nu_{0}=a_{e} \gamma=\frac{a_{e} E}{m_{e} c^{2}}=\frac{E[\mathrm{MeV}]}{440.6486(1)[\mathrm{MeV}]},
$$

where $m_{e}$ is the mass of the electron [10] and $c$ is the speed of light.

Any non-vertical magnetic fields reduce the equilibrium degree of polarization below its maximum of $92 \%$ and perturb the spin precession. A special procedure [11] has been commissioned to establish polarized beams for energy calibration in LEP. The degree of vertical polarization is measured using a Compton polarimeter [11, 12] and was typically $10-20 \%$ during energy calibration at end of coast. Polarizations in excess of $50 \%$ have been observed in dedicated experiments.

The precession frequency of the polarization vector is precisely measured by inducing a resonant depolarization of the beam with a radial oscillating field from a coil. If the perturbation from the radial field is in phase with the spin precession then the spin rotations about the radial direction add up coherently from turn to turn. About $10^{4}$ turns $(\approx 1$ second) are needed to bring the polarization vector into the radial plane. One resonance condition between the perturbing radial field and the nominal spin precession is $f_{\text {dep }}=[\nu] \cdot f_{\text {rev }}$, where $f_{\text {dep }}$ is the frequency of the oscillating field, $f_{\text {rev }}$ is the revolution frequency of the particles, which is precisely known, and $[\nu]$ denotes the non-integer part of the spin tune. Its integer part is known accurately enough from the setting of the bending field.

The frequency of the perturbing field is varied slowly with time over a given frequency range. The difference, $\Delta \nu_{\text {scan }}$, in frequency between the start and the end of the "sweep" determines the resolution of the spin tune measurement and is chosen to be small enough to match the requirements of precise energy calibration. For standard energy calibrations $\Delta \nu_{\text {scan }}$ was set to 0.002 , which corresponds to $0.9 \mathrm{MeV}$ in beam energy. An example of energy calibration by resonant depolarization is shown in figure 1 . Since the depolarization process occurs slowly compared to the periods of the betatron and synchrotron oscillations of the beam particles, the measured average beam energy is, to a very good approximation, independent of these oscillations. It can therefore be determined much more precisely than the beam energy spread $(\simeq 39 \mathrm{MeV})$. Local energy variations, such as the energy loss in the arcs, change the local spin phase advance but they do not bias the measured beam energy, which is determined from the total spin phase advance over one full turn.

To determine the average beam energy uniquely, two additional measurements are required to remove ambiguities inherent in the method [2]. A single depolarization cannot determine whether the spin tune is below or above the half integer: this ambiguity is solved by increasing the beam energy with an RF-frequency change and by measuring the direction of the change in the measured spin tune. Moreover, since depolarization can also occur on synchrotron oscillation satellites of the spin tune, the stability of the tune measurement against a change of the synchrotron tune has to be verified. 


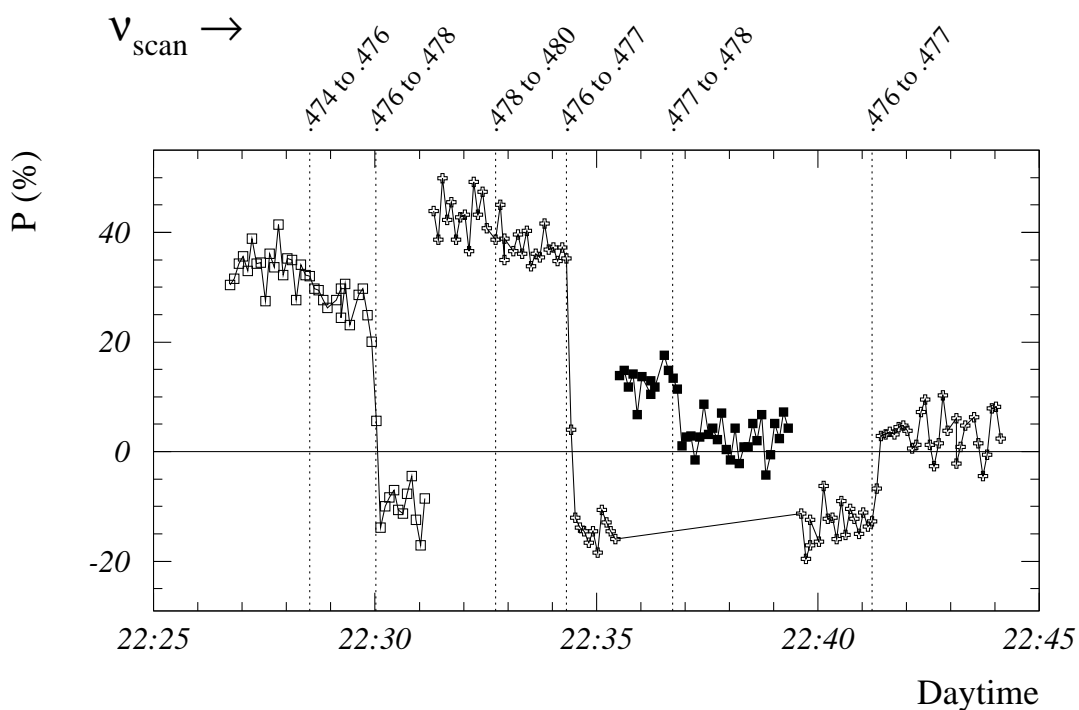

Figure 1: Example of energy calibration. Resonant depolarization is performed on several bunches. Vertical dotted lines indicate the frequency sweeps of the of the radial oscillating field. Partial spin flip to negative polarization was observed and checked by flipping it again.

The total systematic error on a single beam energy measurement by resonant depolarization is about $200 \mathrm{keV}$ as shown by dedicated experimental and theoretical studies [2]. This result is supported by the excellent reproducibility and short-term stability of the measured energy. The systematic error given above contains several theoretical estimates. These could be verified experimentally with only limited precision, as summarized in table 1 , and an experimental upper bound for the systematic error of $1.1 \mathrm{MeV}$, at a beam energy of $45 \mathrm{GeV}$, was established. The most important sources of systematic errors are discussed below.

\begin{tabular}{|l|c|c|}
\hline Source & $\begin{array}{c}\Delta E(E=45.6 \mathrm{GeV}) \\
\text { Theoretical estimates }\end{array}$ & $\begin{array}{c}\Delta E(E=45.6 \mathrm{GeV}) \\
\text { Experimental upper bound }\end{array}$ \\
\hline \hline Electron mass & & $13 \mathrm{keV}$ \\
\hline Revolution frequency $f_{\text {rev }}$ & & $0 \mathrm{keV}$ \\
\hline Frequency of the RF magnet $f_{\text {dep }}$ & & $100 \mathrm{keV}$ \\
\hline Width of excited resonance & & $100 \mathrm{keV}$ \\
\hline Interference of resonances & & $<100 \mathrm{keV}$ \\
\hline Quadratic nonlinearities & $<5 \mathrm{keV}$ & $<500 \mathrm{keV}$ \\
\hline Spin tune shifts from long. fields & $<5 \mathrm{keV}$ & $<500 \mathrm{keV}$ \\
\hline Spin tune shifts from rad. fields & $<100 \mathrm{keV}$ & $<800 \mathrm{keV}$ \\
\hline \hline Total systematic error & $200 \mathrm{keV}$ & \\
\hline \hline Total upper bound & & $1.1 \mathrm{MeV}$ \\
\hline
\end{tabular}

Table 1: This table summarizes the systematic errors on the measurement of the beam energy by resonant depolarization. A standard energy calibration with a well corrected vertical closed orbit is assumed. All errors are understood to be Gaussian and refer to the energy of a single beam. The contributions shown in the third column are experimental upper bounds and are used to compute the total upper bound on the systematic error. The total systematic error is computed using the theoretical estimates for the last three entries of the table. 


\subsection{Interference between resonances}

It was suggested in [13] that interference between the artificially excited spin resonance and "natural" spin resonances could result in a shift of the measured spin tune. The measured beam energy would then be biased. The effect was studied experimentally by approaching strong natural spin resonances. The beam energy was changed by setting the RF-frequency to different values. For each setting the beam energy was measured by resonant depolarization. Any significant shift due to interference would disturb the expected linear dependence of the beam energy, $E$, on the RF-frequency, $f_{\mathrm{RF}}$, when approaching strong spin resonances. No such effect was seen (see figure 2). From the experimental results any bias of standard energy calibrations due to interference of spin resonances can be excluded down to $\Delta E / E=2 \times 10^{-6}$.

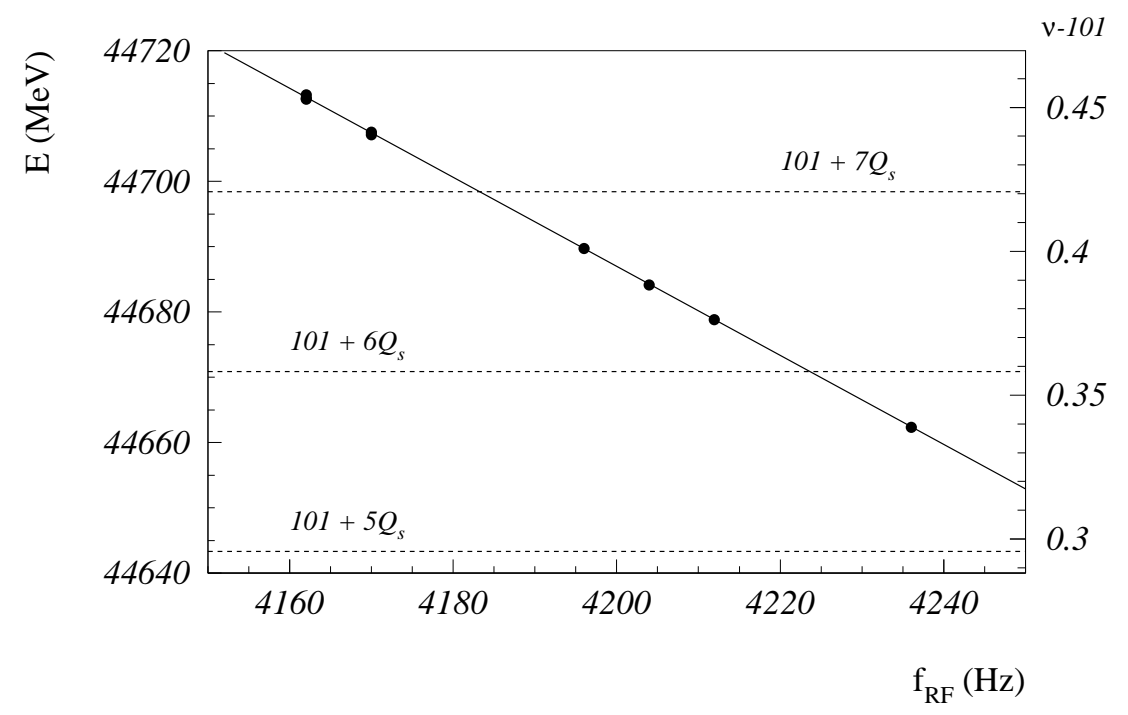

Figure 2: Measured change of beam energy, $E$, as a function of the RF frequency, $f_{R F}$. Only the last four digits of $f_{R F}$ are indicated (nominal $f_{R F}=352254170 \mathrm{~Hz}$ ). During the measurement several strong spin resonances, indicated by the horizontal dotted lines, were approached but no unexpected shifts of the beam energy were observed. From this experiment the momentum compaction factor, $\alpha_{c}$, is determined to be $(1.86 \pm 0.02) \times 10^{-4}$, which compares very well with the calculated value of $1.859 \times 10^{-4}$

\subsection{Quadratic nonlinearities}

Small systematic shifts of the spin tune can occur due to the spin tune spread related to synchrotron oscillations of the individual particles. This effect is expected to be very small. It was shown that the spin frequency spread does not exceed the value $10^{-5} \cdot f_{\text {rev }}[14]$. For LEP this corresponds to a relative error of $\Delta E / E<1 \times 10^{-7}$, or to about $5 \mathrm{keV}$ at $45 \mathrm{GeV}$ beam energy. The effect is modified by variation of quadratic nonlinearities, e.g. the chromaticity of radial betatron oscillations. To check for this effect the chromaticity was changed and the spin tune was remeasured. No significant effect was observed, within a resolution of $0.5 \mathrm{MeV}$, when the chromaticity was increased by +10 .

\subsection{Spin tune shifts due to non-vertical magnetic fields}

Non-vertical magnetic fields can modify the simple relationship (equation 3 ) between spin tune and beam energy by a small amount. 
In LEP, strong longitudinal fields arise from the experimental solenoids and radial fields occur mainly due to vertical closed orbit deviations at the quadrupoles. Since threedimensional rotations do not commute, small spin tune shifts would directly result in a bias of the energy calibration.

The effect was studied theoretically for the experimental solenoids and was found to be small [15]. Near the operating spin tune, the spin tune shift due to the solenoids produces an energy bias smaller than $50 \mathrm{keV}$, without spin matching [11] of the solenoids, and smaller than $5 \mathrm{keV}$ with spin matching. This prediction was tested experimentally by switching off the spin matching bumps for the solenoids after an energy calibration and measuring the spin tune again. No change was observed within the resolution of the measurements.

Radial magnetic fields caused by random misalignment of the quadrupoles can cause spin tune shifts. Numerical calculations [16] have shown that near the operating spin tune the largest bias comes from the spread of possible spin tune shifts for different imperfections. This bias is $30 \mathrm{keV}$ for an RMS vertical orbit of $0.5 \mathrm{~mm}$ and smaller than $100 \mathrm{keV}$ in all practical cases. Different vertical orbits sample the radial magnetic fields in a different way and therefore a change in the vertical orbit can cause additional spin tune shift. This effect has been investigated experimentally by looking at spin tune changes after vertical corrections were made. Out of two dozen cases two significant changes were observed: their extent was in one case between 0.4 and $1.2 \mathrm{MeV}$ on the beam energy, and in the other between 0.1 and $0.5 \mathrm{MeV}$, the uncertainty being due to the resolution of the measurements. We take the centre of the larger change as implying a conservative upper limit of $800 \mathrm{keV}$ for the effects caused by vertical orbit distortions.

\section{Energy of the positron beam}

Imperfections of the LEP lattice, such as misalignment of quadrupoles, imperfections in the fields of quadrupoles and sextupoles, asymmetries in the optical sequence and the pretzel scheme, combined with the sawtooth effect (see fig. 3) can cause horizontal deviations from the ideal orbit which are different for electrons and positrons. These produce unequal average energies for the two beams. Theoretical arguments suggest that this difference is smaller than $0.3 \mathrm{MeV}$ [17].

The polarimeter is normally configured to measure the polarization of only the electron beam, so energy calibration by resonant depolarization is usually performed just for electrons. One special calibration was performed in 1993, and two in 1994, to measure the electron and positron beam energies as close to simultaneously as possible. The 1993 calibration indicated an energy difference larger than $0.5 \mathrm{MeV}$ [2]. The two 1994 calibrations found that the positron beam was $0.0 \pm 0.2 \mathrm{MeV}$ and $0.4 \pm 0.4 \mathrm{MeV}$ higher in energy than the electron beam.

We include a correction of $+0.3 \mathrm{MeV}$ to the energy of the positron beam with respect to the electron beam, and assign a systematic error of $0.3 \mathrm{MeV}$. This error covers all three of the measurements made.

\section{$3 \quad$ Beam energy at the interaction points}

The energy of the beam is not constant as it goes around the LEP ring. The energy loss of about $125 \mathrm{MeV}$ per turn of electrons and positrons on their curved path through the dipoles in the LEP arcs is compensated by acceleration in the RF cavities placed either side of the L3 and OPAL interaction points, which are diametrically opposite each other across LEP. 

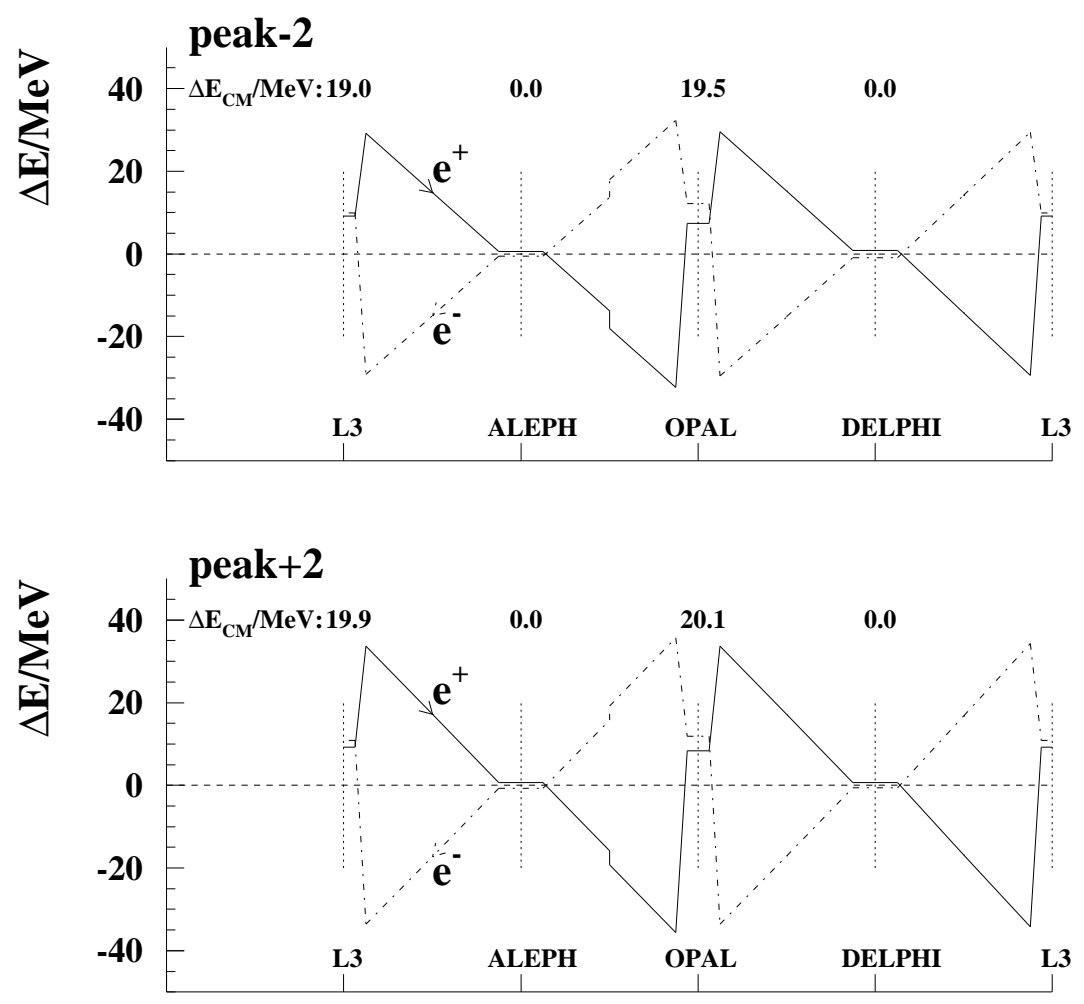

Figure 3: Deviations from the mean energy in the LEP arcs of electrons and positrons (sawtooth effect) for average 1993 running conditions at the peak-2 and peak +2 energy points. The step between the ALEPH and OPAL interaction points is due to emittance wigglers which were in operation at the beginning of fills in order to limit beam-beam effects by increasing the bunch emittance.

The deviation of the beam energy from the mean energy as a function of the position around the ring is shown in figure 3, for the average running conditions during 1993.

Ideally, the positioning of the RF units matches the frequency at which they are operated and, in the absence of alignment errors, the sum of the energies of the electron and positron beams would be constant around the whole ring.

Due to a difference between the design frequency $f_{1}$, used to position the RF cavities, and the actual frequency used during physics running $f_{R F}$, the beam energies at the OPAL and L3 interaction points are higher by about $10 \mathrm{MeV}$, leading to a centre of mass energy at these interaction points which is about $20 \mathrm{MeV}$ higher than the average energy in the LEP arcs [7]. Should one or more RF units be running at an atypical setting, or should there be a difference in path length for particles going from L3 (interaction point 2) to OPAL (point 6) via ALEPH (point 4) or via DELPHI (point 8), then such offsets can arise also at the DELPHI and ALEPH interaction points.

The difference between the two frequencies $f_{1}$ and $f_{R F}$ causes an average phase error $\phi_{e}:$

$$
\phi_{e}=\frac{\left(f_{1}-f_{R F}\right) d}{c} \times 360^{\circ} \simeq-10^{\circ}
$$

where $d=196.41 \mathrm{~m}$ is the distance of the effective centre of the RF units from the interaction points and $c$ is the speed of light. The energy offsets per beam at the interaction 
point can be calculated from the difference in energy gain on the incoming side and on the outgoing side, which are given by the accelerating voltage, $U_{R F}$, and the phase angles,

$$
\Delta E_{b}^{I P}=\frac{1}{2}\left(U_{R F}^{i n} \sin \left(\phi_{1}+\phi_{e}\right)-U_{R F}^{\text {out }} \sin \left(\phi_{1}-\phi_{e}\right)\right) .
$$

In this formula $\phi_{1}$ is the stable phase angle of the bunches with respect to $f_{1}$. Its value was about $150^{\circ}$ during the 1993 running period.

In practice, a more detailed model was used to calculate the energy offsets in order to account for variations of the RF voltages with time, changes of the energy loss per turn caused by the use of the emittance wigglers during physics running, and an effective reduction of the accelerating voltage in the RF cavities due to the energy taken out of the cavities by the bunches. The last effect introduces a small dependence of the energy correction on the beam currents. The model was also used to evaluate systematic errors on the correction arising from the errors in the positions of the RF cavities and the calibration and precision of the phases of the RF voltages. In the simulation, the average phase angle of each beam, $\phi_{s}^{ \pm}$, is determined by setting equal the energy loss per turn and the energy gain in the RF cavities. The energy offsets were provided to the experiments for each 15 minute period during the physics running, based on the RF voltages, wiggler and beam currents logged for that period. The information on cavity positions needed for this calculation is obtained from two sources. The short distances between the cavities around a single experiment (L3 or OPAL) were obtained by direct optical surveys of their positions, with a precision of approximately $1 \mathrm{~mm}$. The distances between the cavities at L3 and OPAL (i.e. the lengths of the two half-rings between these points) were measured using the RF system itself, by comparing the cavity phase settings needed to maximize the energy transfer to the two counter-rotating beams. This showed that the two half-rings were equal in length to within $5 \mathrm{~mm}$. This method was also used to confirm the optical alignment around L3 and OPAL, again with a precision of about $5 \mathrm{~mm}$.

The model is used also to compute the synchrotron tune for each beam, $\mathrm{Q}_{\mathrm{s}}^{ \pm}$. Knowing the phase angles in each cavity, $\phi_{i}^{ \pm}$, the values $\mathrm{Q}_{\mathrm{s}}^{ \pm}$are calculated from the sums of the derivatives of the energy gain, $\sum d E_{i} / d \phi_{i}^{ \pm}$,

$$
\mathrm{Q}_{\mathrm{s}}^{ \pm}=\sqrt{\frac{-h \alpha_{c} \sum d E_{i} / d \phi_{i}^{ \pm}}{2 \pi E_{\text {beam }}}}
$$

Here, $h=31324$ is the harmonic number of LEP, and $\alpha_{c}=1.86 \times 10^{-4}$ is the momentum compaction factor of the LEP lattice. A comparison with the measured value of $\mathrm{Q}_{\mathrm{s}}$ provides an important cross check on the consistency of the input parameters needed to calculate $\Delta E_{b}^{I P}$.

During LEP operation in 1993, the measured and the calculated values of the synchrotron tune, $\mathrm{Q}_{\mathrm{s}}^{\text {meas }}$ and $\mathrm{Q}_{\mathrm{s}}^{\text {calc }}$ respectively, were compared on a regular basis. Any problems with either the RF system or the logging of its parameters could be detected and fixed before they significantly affected off-peak running. The value of the phase of the RF voltages was not measured regularly and therefore unexpectedly low values of $\mathrm{Q}_{\mathrm{s}}^{\text {meas }}$ were taken as an indication of the presence of phase errors and the RF system was rephased. A large difference between the synchrotron tune of electrons and positrons would also provide an indication of significant deviations in the voltage phasing.

The model is also used to compute the shift of the longitudinal position of the collision point due to an asymmetric distribution of power over the RF units. No inconsistency 
was found comparing the prediction of the model with the measured vertex position using ALEPH data.

\subsection{Evaluation of errors in the RF correction}

The dominant systematic error on the RF correction arises from uncertainties in the precise positions of the RF cavities. The uncertainty in the measured lengths of the LEP arcs corresponds to an uncertainty of $\pm 1 \mathrm{MeV}$ in centre of mass energy at points 4 and 8 , which is anti-correlated between the two points. Because of the smallness of the energy correction at points 4 and 8 , this is the only significant error at these interaction points. The uncertainties on the distance between the cavities around L3 or OPAL produce an uncertainty of $\pm 0.4 \mathrm{MeV}$ in the centre of mass energy at points 2 and 6 , uncorrelated between the two points. Systematic errors on the energy correction arising from uncertainties in the input parameters to the model, and from imperfections of the model itself, were investigated by comparing the measured and the calculated values of the synchrotron tune. The average of the measured $Q_{s}$ values during the 1993 energy scan was 0.065 , with an RMS spread of 0.002 . The difference between the calculated and the measured $Q_{\mathrm{s}}$ had a Gaussian shape with an RMS spread of only about 0.0005 (see figure 4). There was also good agreement between the synchrotron tunes of electrons and positrons, which showed an average difference of only 0.0001. Possible constant errors in the voltage phasing between points 2 and 6 were estimated to be below $\pm 5^{\circ}$ and give rise to an error of $\pm 0.5 \mathrm{MeV}$ for points 2 and 6 . This error is anti-correlated between the two
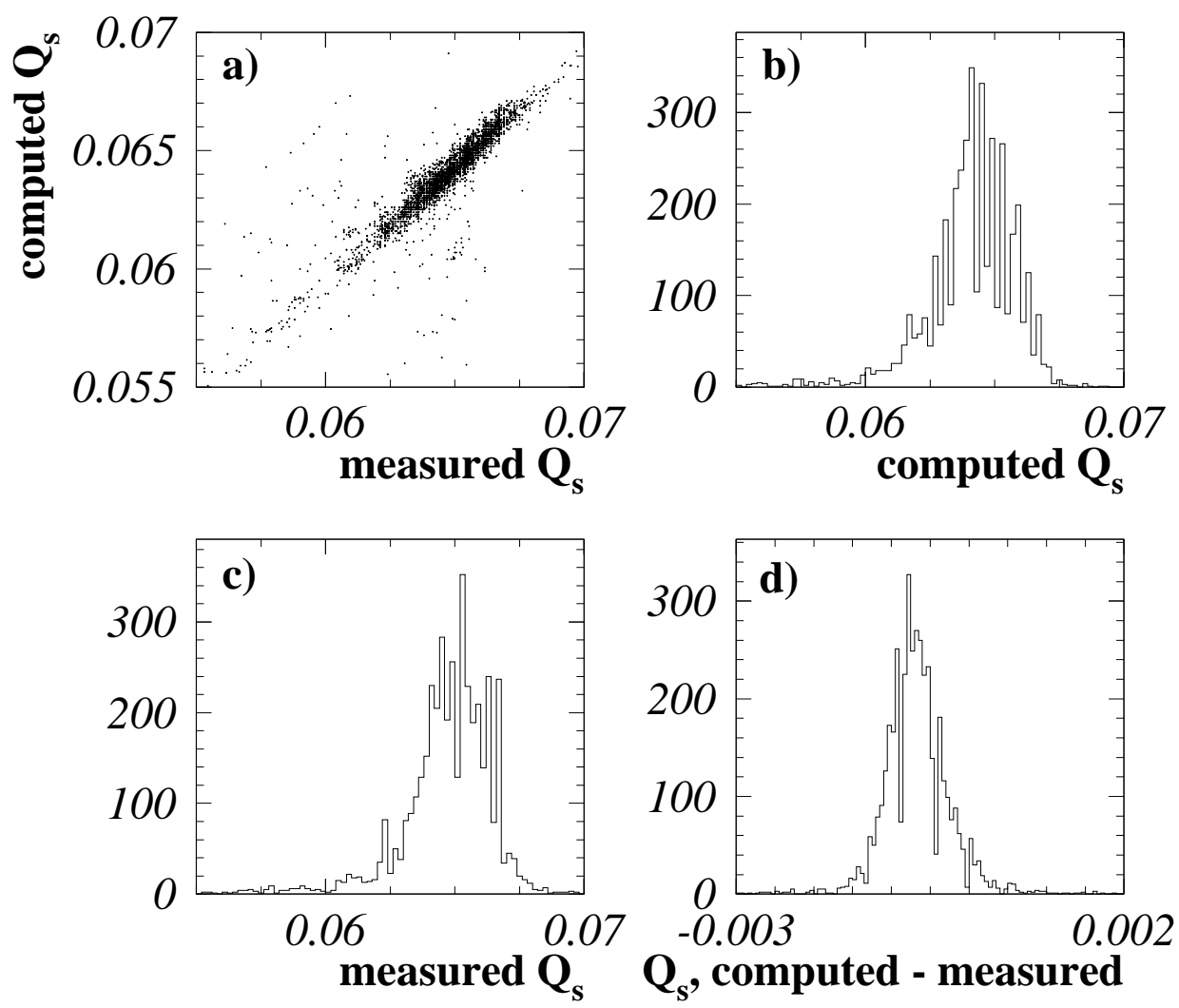

Figure 4: Comparison of the measured value of the synchrotron tune, $\mathrm{Q}_{\mathrm{s}}$, and the value computed from various parameters (see text). The sidebands in a), corresponding to $\left|\mathrm{Q}_{\mathrm{s}}^{\text {meas }}-\mathrm{Q}_{\mathrm{s}}^{\text {calc }}\right| \simeq 0.004$, are due to different times of logging of the parameters. The shift away from zero in $d$ ) was used to adjust the overall calibration of the RF voltages. 
interaction points.

The average difference of 0.0007 between the measured and the calculated $Q_{s}$ corresponds to a $1.5 \%$ shift in the calibration of the RF voltages. An uncertainty of $\pm 1 \%$ was assumed on this calibration, resulting in a correlated error of $\pm 0.2 \mathrm{MeV}$ on the centre of mass energies in points 2 and 6 . This error takes into account all effects which lead to a broadening of the distribution of the difference between the measured and the calculated $\mathrm{Q}_{\mathrm{s}}$, in particular the resolution of the $\mathrm{Q}_{\mathrm{s}}$ measurements, the measurements of the RF voltages and possible time-dependent variations of the $R F$ phasing.

After this voltage calibration, the difference between $Q_{s}^{\text {calc }}$ and $Q_{s}^{\text {meas }}$ was found to be close to zero at all three energy points. The difference between the peak +2 and the peak -2 points was 0.0002 and corresponds to a difference in energy of $\pm 0.1 \mathrm{MeV}$ at points 2 and 6 . The uncertainty in the momentum compaction factor, measured to be $(1.86 \pm 0.02) \times 10^{-4}$, results in an error of $\pm 0.2 \mathrm{MeV}$ on the centre of mass energy at interaction points 2 and 6 and is correlated between them. There is a residual dependence on the bunch current in the difference between the calculated and measured values of $\mathrm{Q}_{\mathrm{s}}$, which causes a correlated energy error of at most $\pm 0.2 \mathrm{MeV}$ on the energy in points 2 and 6.

Combining the various sources results in an uncertainty of $\pm 0.6 \mathrm{MeV}$ at point 2 and 6 , which is almost uncorrelated between the two points.

\section{$4 \quad$ Model of the energy variation}

The typical time variation of the LEP energy is of the order of $1 \mathrm{MeV}$ per hour. Individual experiments have slightly different efficiencies and integrated luminosities within a fill. The energy of LEP is calculated in 15 minute intervals for each interaction point for every physics fill, following a model which will be discussed in this section.

The mean energy of electrons and positrons revolving around the LEP ring is determined by the magnetic fields they encounter in their closed orbit. The integral of the magnetic field seen by the particles in the ring has two main contributions: one comes from the dipole fields of the bending magnets around the arcs and the other from higher order magnetic fields, mainly arising from the quadruple focusing and defocusing magnets.

\subsection{Dipole fields}

Great care was taken to stabilize and to monitor the magnetic field of the LEP dipoles during the scan. The dipole current was kept stable to better than 3 parts in $10^{5}$ and was regularly monitored. The temperature of the magnets was also kept stable (the RMS variation with time was about $0.2{ }^{\circ} \mathrm{C}$ ) since it was known to influence both the effective length of the magnet and the magnetic field [7] causing a fractional change of the energy of $0.0001 /{ }^{\circ} \mathrm{C}$. It was monitored by measuring the temperature of 34 magnets inside the tunnel.

The magnetic field was monitored using an NMR probe measuring the field in a reference magnet, connected in series with the LEP dipoles but mounted outside the tunnel. This reference magnet is intended to exhibit the same hysteresis effects as the LEP dipoles.

Occasional jumps are observed in the field of this magnet (see figure 5). Their magnitude is typically equivalent to a few $\mathrm{MeV}$ in beam energy. Many of these jumps have been correlated with small, transient flux changes in the flux loop system (see section 6). It is possible that the jumps are caused by transient defects in the $\mathrm{AC}$ power supplied to CERN. These are not fully attenuated by the power converters and appear as rapid 

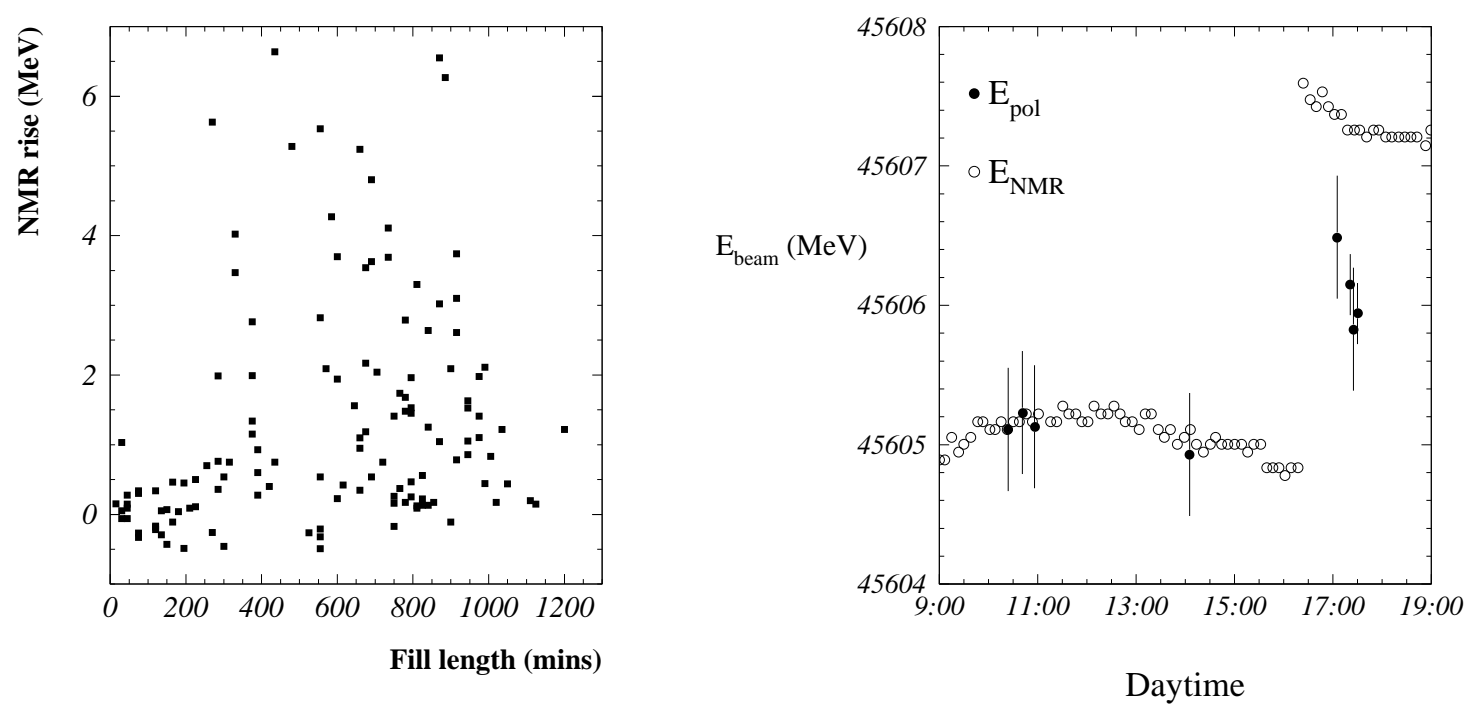

Figure 5: Left: Rise observed in the reference magnet field as a function of fill length. There are two populations visible - those fills which contained a jump in the field, scattered across the upper part of the figure, and those which did not. The field rise of the lower group shows some evidence of being correlated with length of fill. The field readings from the NMR probe have been converted to equivalent beam energies for convenience. Right: A jump in the NMR readings observed during an energy calibration. The points with error bars are the LEP energies measured with resonant depolarization, while the other points are the LEP energy predicted from the field in the reference magnet. Approximately $50 \%$ of the rise seen in the NMR is visible in the true beam energy.

excursions in the dipole current. The hysteresis of the dipole magnet iron could then result in a net change of the magnetic field that remains for the rest of the fill. Artificial transients have been induced in the power converters and behaviour similar to the jumps has been observed. The detailed response of the field depends on the duration, waveform and amplitude of the transients, all of which are generally unknown, so it is difficult to compare quantitatively the artificial jumps with the real ones.

In particular, the field at the beam position might not have as large a change as that recorded by the NMR probe. This can be seen in figure 5 which shows a jump in the NMR reading observed during an energy calibration. Only $50 \%$ of the field increase recorded by the NMR probe seems to be present at the beam position. It is thought that this difference is due to the absence of a beam pipe surrounding the NMR probe in the reference magnet. Although the non-magnetic beam pipe should have no effect on measurements of the DC field, the rapidly changing incremental field lines during a transient event are partially excluded from the beam position by eddy currents in the pipe. When the transient is over, these field lines are "frozen" into position by the hysteresis of the magnet iron, away from the beam position. This theory has been supported by an experiment performed in 1994 , when a second NMR probe inside a beam pipe was installed in the reference magnet. The jumps measured by this second NMR correlate in time with those measured by the first NMR, but the amplitude measured by the NMR in the beam pipe is generally about half.

The second and most important effect visible in the NMR readings is a slow rise over the course of a fill. It is not known what causes this. It is observed to be correlated with the temperature rise in the dipoles in the LEP tunnel, although not with the temperature 
rise of the reference magnet itself. In 1994 it is observed to be $100 \%$ correlated with the measurement of the second NMR installed in a beam pipe in the reference magnet. It is not known whether there is a corresponding rise in the beam energy.

Because the correlation between the increase in the NMR field measurement and the energy can be anywhere from $0 \%$ to $100 \%$, we conservatively include $50 \%$ of the rise as a correction to the calculated energy and use $\pm 50 \%$ of the rise as the error on this correction.

The last dipole field contribution comes from the so called "QFQD compensation coil". LEP is running with significantly different phase advances for the horizontal and vertical planes and there is a net current around the ring arising from the fact that the current required in the focusing quadrupoles is larger than that in the defocusing quadrupoles. To cancel the magnetic fields created by this current asymmetry, a compensating current loop has been installed in the LEP ring. Due to a mistake, this loop was reversed or turned off for some periods of data taking during the scan. The current in the loop was logged during the whole scan. The loop is positioned approximately one metre from the beam path and at the same height, so that the magnetic field due to this current has a vertical component at the beam position. Because of the presence of many shielding objects, such as dipole and quadrupole magnets, it is not possible to calculate accurately the effect on the energy of the beams. The magnitude of the effect was measured directly in a dedicated experiment by comparing the LEP energy, measured by resonant depolarization, before and after inverting the current in the loop. It was found to be $3.0 \pm 1.4 \mathrm{MeV}$ in beam energy for a typical 70 ampere change. The energy model includes a correction based on the logged value of the current in this loop.

\subsection{Quadrupole fields}

A particle going off-centre through a quadrupole magnet sees a magnetic field proportional to its offset. Energy and beam position are therefore related: the energy of LEP changes by about $1 \mathrm{MeV}$ for a $13 \mu \mathrm{m}$ transverse movement of the beam relative to the centre of the quadrupoles.

The lengths of the orbits of the beams are fixed by the frequency of the RF system and a relative movement of the beam with respect to the centre of the quadrupoles can be caused by a change of this frequency or by a change of the mean radius of LEP. The frequency was kept constant during the scan. The mean radius of LEP changes due to ground motion caused by geological and gravitational effects.

The effect of earth tides on the beam energy was demonstrated in a previously published experiment [18] in which the measured energy variation of LEP was correlated with a prediction based on a tidal model. Various models exist which estimate the effect of tides on the circumference of the LEP ring. The model we are using [19] has been checked by a series of dedicated experiments (see figure 6) that have shown a very good agreement between the predictions and the measurements. The relative transverse position of the beam with respect to the quadrupoles can be monitored by the LEP beam orbit monitor (BOM) system [20,21]: a series of capacitive pickups that measure the positions of electrons and positrons with a relative precision of a few microns. The correlation between the beam position measured by the BOM system and the energy predicted by the tide model is shown in figure 7 . The energy difference versus the offset from the quadrupole centre (see figure 7) shows a linear relationship with a slope of $13.0 \pm 0.1 \mu \mathrm{m} / \mathrm{MeV}$, in agreement with the prediction. Since we use the BOM information to estimate beam orbit positions over the whole year, in addition to the above experiment, which demonstrates 
a good short term behaviour, we also need a good fill-to-fill reproducibility of the BOM system. This has been studied through the variations of the beam orbit position in the vertical plane, which are less sensitive to geological movements, and found to be at the $15 \mu \mathrm{m}$ level [21].
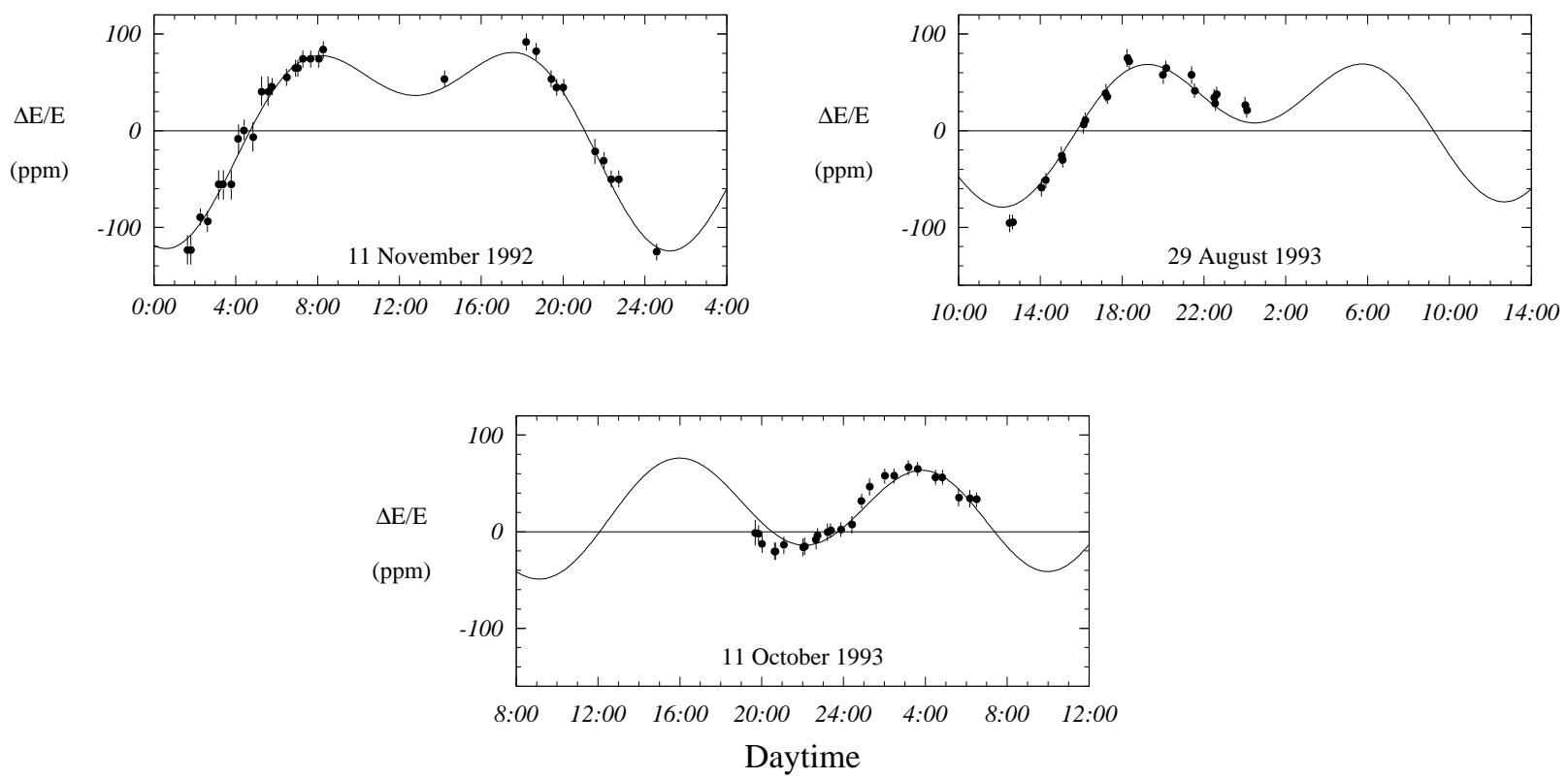

Figure 6: Results of dedicated tide experiments. The beam energy measured with resonant depolarization has been corrected for changes of the integrated dipole field. The agreement between model and measurements is good.

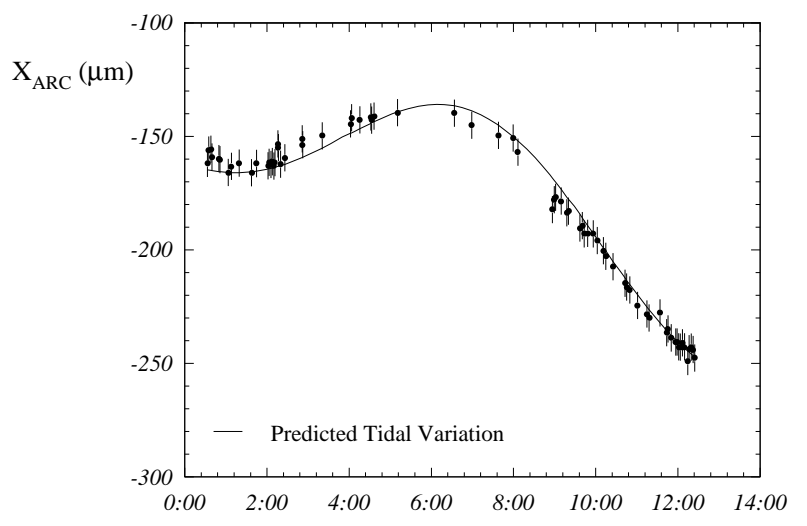

Daytime

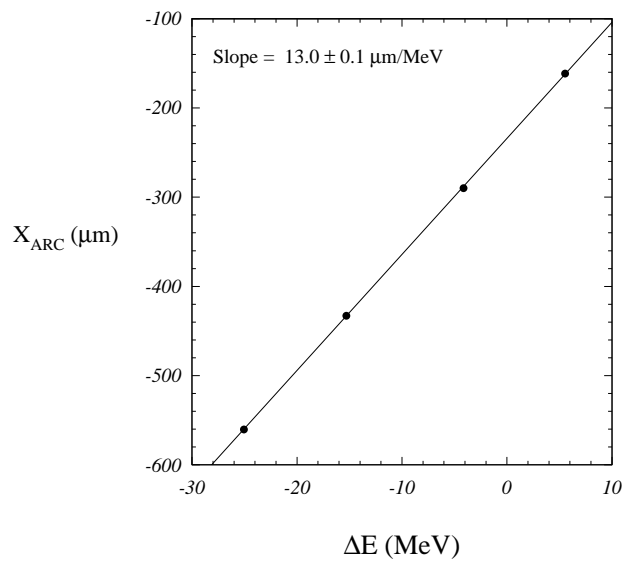

Figure 7: Left: Motion of the beam orbit during a tide experiment. The stability of the BOM readout is better than $5 \mu \mathrm{m}$. Right: Energy variation versus orbit position measured by the BOM system. This is the result of a dedicated experiment which varied the energy by changing the RF frequency. 
The horizontal beam orbit position during the scan is shown in figure 8 , after correction for the effects of tides. There is a rise of the beam energy of about $12 \mathrm{MeV}$ during the last part of the scan. This general trend is also seen in the LEP energy calibration measurements over the whole year, also shown in figure 8. We attribute this to slow geological and hydrological changes in the rock surrounding the LEP tunnel, which cause changes in the circumference of the ring of a few parts in $10^{8}$. By including these effects in the model via the orbit information, we reduce the scatter of the calibration data with respect to the model prediction by about a factor of two.

\subsection{The model}

The energy variation of LEP, as a function of the time $t$ from the start of the fill, is computed according to the following formula:

$$
\begin{aligned}
E_{\text {lep }}= & C_{\text {norm }}(\text { fill }) \times\left(E_{N M R}(t=0)+\frac{E_{N M R}(t)-E_{N M R}(t=0)}{2}\right) \times\left(1+C_{\text {tdipole }}(t)\right) \\
& \times\left(1+C_{\text {tide }}(t)\right) \times\left(1+C_{\text {orbit }}(\text { fill })\right) \times\left(1+C_{\text {c. coil }}(t)\right) \\
& \times\left(1+C_{R F}(t)\right) \times\left(1+C_{\text {tref }}(t)\right)
\end{aligned}
$$

In this formula $C_{\text {norm }}$ is used for absolute normalization, whereas all other terms follow the relative energy changes. The individual effects have been discussed previously and the meaning of each of the terms is explained below.

- $C_{\text {norm }}$ is different depending on whether the fill in question was calibrated using the resonant depolarization method or not. If it was, then this normalization factor ensures that the energy of the model at the moment of the calibration equals the value of the calibration of this fill. If it was not, this term is equal to the mean normalization factor of all the calibrated fills at that energy point.

- The term involving $E_{N M R}$ at the start of the fill $(t=0)$ corrects for hysteresis effects. The difference between $E_{N M R}$ at the current time, $t$, and at the start of the fill takes into account the uncertainty in the magnetic monitoring.

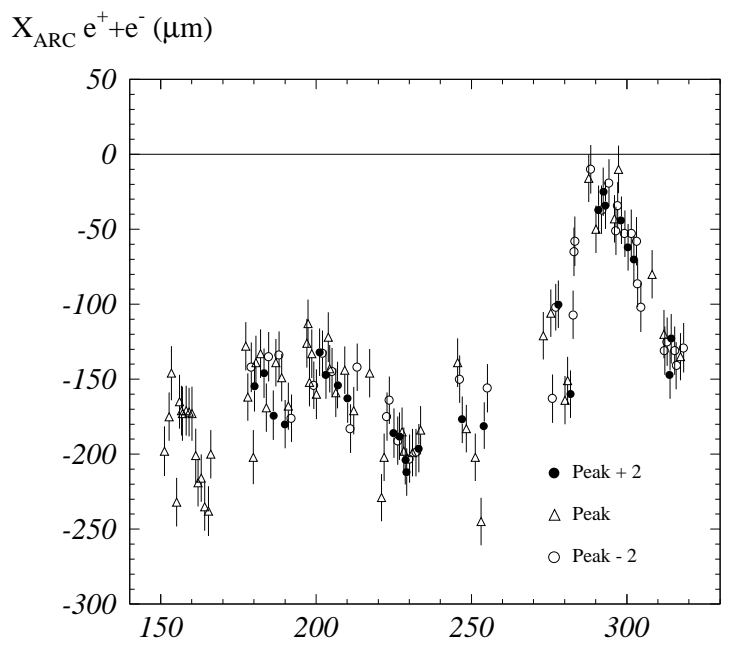

Time (days)

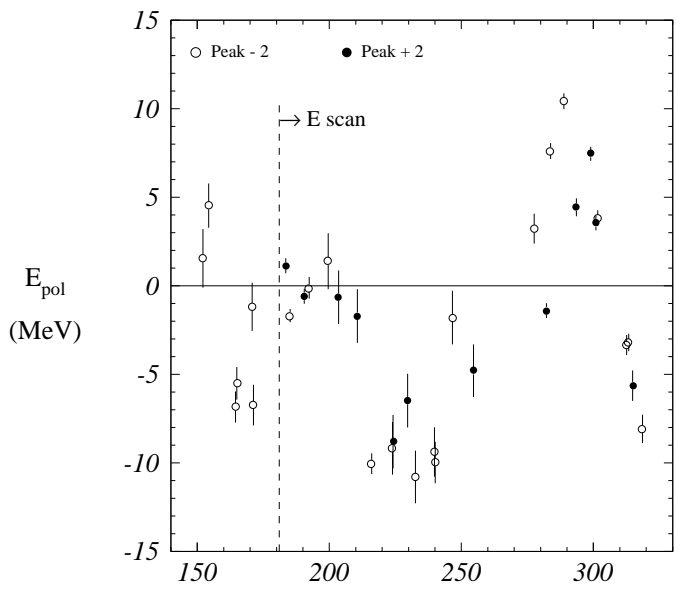

Time (days)

Figure 8: Left: Mean X position of the beam versus time in 1993 Right: Average beam energy measured by resonant depolarization versus time in 1993. The energy has been corrected for changes in the integrated dipole field and for tidal effects. A constant offset has been subtracted from the data at each scan point in order to allow both sets to be plotted on the same figure. 
- $C_{\text {tdipole }}$ is the temperature correction for the main dipole magnets.

- $\quad C_{\text {tide }}$ is the correction due to the effect of the tide [19].

- $C_{\text {orbit }}$ is the correction for the horizontal position of the orbit. This effect is calculated using an average orbit position for each fill after the expected variations for tide have been removed.

- $C_{c \text { coil }}$ is the correction for current in the QFQD compensation coil.

- $C_{R F}$ is the correction, different for each interaction point, due to the RF system.

- $\quad C_{\text {tref }}$ is the temperature correction for the reference magnet. The temperature of the reference magnet is well controlled, and it has by construction a factor of 10 lower sensitivity to temperature than the main dipoles.

All the corrections, with the exception of $C_{\text {norm }}$ and $C_{\text {orbit }}$, are applied according to the conditions at that particular time, whereas $C_{\text {norm }}$ and $C_{\text {orbit }}$ are applied on a fill-byfill basis. This model of the energy variation has been used to compute the luminosityweighted mean centre of mass energy of the fills at each scan point for each experiment.

\section{$5 \quad$ Systematic errors on the energy at each scan point}

The luminosity-weighted energies at the interaction points are affected by systematic errors inherent to the measurement of the beam energy with resonant depolarization (presented in section 1), to the uncertainty on the positron beam energy (presented in section 2) and to the corrections to convert the average beam energy to the energy at the interaction points (presented in section 3 ).

The energies of the calibrated fills are precisely measured at the ends of the fills, using resonant depolarization. Other systematic errors arise only to the extent that these energy measurements are not representative of the conditions earlier in the fills, during physics running. The mean energy of the uncalibrated fills is estimated from the calibrated ones on a statistical basis and additional systematic errors arise from the precision in evaluating this mean energy using the calibration measurements.

The various contributions to the systematic error on the off-peak energies are discussed in the following and are summarized in table 2. To a good approximation, the systematic errors on $\mathrm{M}_{\mathrm{Z}}$ and $\Gamma_{\mathrm{Z}}$ arising from the LEP energy calibration are related to the energy errors by equations 1 and 2 . When the energy errors at the two off-peak points have approximately the same size, they can also be separated in terms of the errors that are correlated and uncorrelated between the two points. In that framework, the error on $\mathrm{M}_{\mathrm{Z}}$ is given by the correlated error plus the uncorrelated part divided by $\sqrt{2}$, while the error on $\Gamma_{\mathrm{Z}}$ is approximately given by the uncorrelated error, since the numerical coefficient in equation 2 is approximately equal to $1 / \sqrt{2}$. The correlated and uncorrelated errors are also shown in table 2 when applicable.

\subsection{Mean fill energy}

To the extent that the energy calibration measurements are, in the statistical sense, an unbiased sample of the energies of the electrons under physics conditions, we can use them to form an estimate of the mean energy and the RMS variation about that mean. The use of a model for the energy variation over time complicates this only a little: the mean energy is used as a global normalization factor for the model $\left(C_{\text {norm }}\right.$ in equation 7 ) and the deviations are computed with respect to the prediction of the model.

The distribution of the deviations is shown in figure 9 . If the model were to describe all aspects of the time dependence of the energy, the distribution of the deviations should 


\begin{tabular}{|l||r|r|r||r|r|}
\hline Source & peak-2 & peak+2 & Correlation & $\begin{array}{r}\text { Correlated } \\
\text { Error }\end{array}$ & $\begin{array}{r}\text { Uncorrelated } \\
\text { Error }\end{array}$ \\
\hline \hline Mean fill energy & 1.60 & 0.72 & 0 & NA & NA \\
\hline Temperature Correction & 0.16 & 0.35 & 1.00 & NA & NA \\
\hline QFQD Correction & 0.40 & 0.36 & 1.00 & 0.4 & 0 \\
\hline Tide & 0.04 & 0.41 & 1.00 & NA & NA \\
\hline NMR Uncertainty & 0.93 & 0.93 & 0.81 & 0.8 & 0.4 \\
\hline Energy Measurement & 0.50 & 0.50 & 0.04 & 0.1 & 0.5 \\
\hline $\mathrm{e}^{+}$Energy Uncertainty & 0.30 & 0.30 & 0.5 & 0.2 & 0.2 \\
\hline \hline RF Corrections (Pt. 2 or 6) & 0.60 & 0.60 & 0.97 & 0.6 & 0.1 \\
\hline RF Corrections (Pt. 4 or 8) & 1.02 & 1.02 & 0.96 & 1.0 & 0.1 \\
\hline RF Corrections (Combined) & 0.22 & 0.22 & 0.90 & 0.2 & 0.0 \\
\hline
\end{tabular}

Table 2: Summary of errors (MeV) on the energy determination. These numbers are for illustration only - the exact errors are determined using the correlation matrix formalism. The first three columns give the errors at peak -2 and peak +2 and their correlation coefficient. The last two columns give the correlated and uncorrelated error as explained in the text. NA= Not Applicable. The errors summarized in the upper part of the table apply to the energies at each interaction point. The errors described in the lower part of the table apply, respectively, to the energies at interaction points 2 or 6 (L3 or OPAL), points 4 or 8 (ALEPH or DELPHI), and to the energies averaged among the four interaction points.

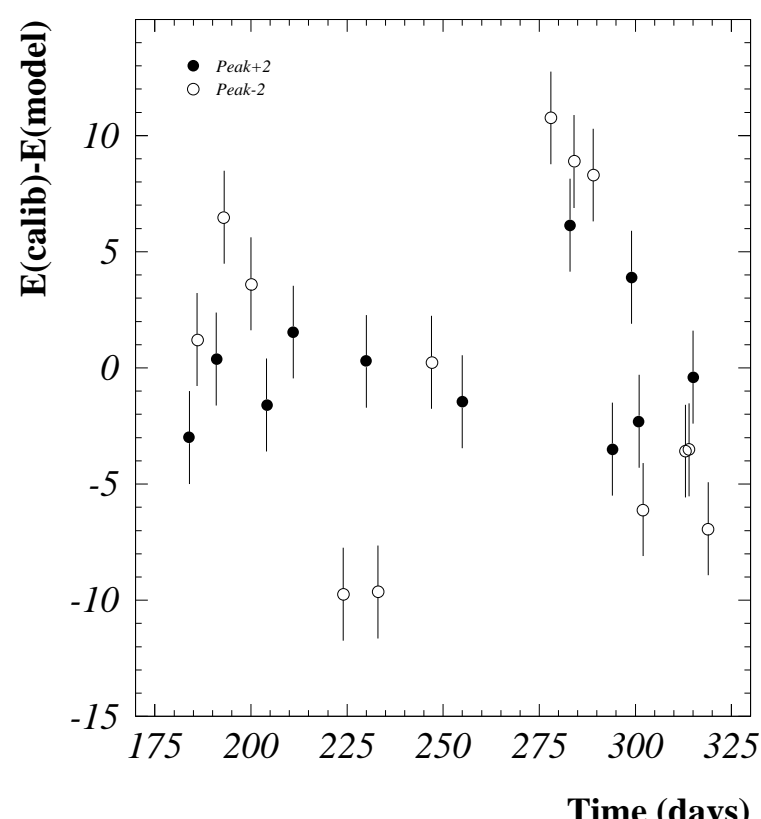

Figure 9: Difference between the centre of mass energies measured during the scan by the resonant depolarization method and those predicted by the model with a constant normalization factor. An error bar of $2 \mathrm{MeV}$ is shown on each entry. 
reflect the combined statistical precision of the measurements and of the parameters of the model.

The RMS scatters are respectively 2.8 and $6.9 \mathrm{MeV}$ for the centre of mass energies of the peak +2 and peak-2 fills. As there are 11 and 13 energy measurements using the resonant depolarization method in physics fills for the two points, the probability that the two observed distributions are statistically compatible with having come from a single distribution with an RMS of $5.4 \mathrm{MeV}$ is about $1 \%$. Studies of the uncertainties of the energy measurements and of the parameters used for the model, described previously, indicate that less than $2 \mathrm{MeV}$ of this scatter can be attributed to these sources, so we attribute all the scatter to real variations of the LEP energy due to unknown effects.

The error on the average energy of the uncalibrated fills then arises from two types of fluctuations. Firstly, we use a finite number of measurements to estimate the mean energy, so that estimate can be in error by

$$
\frac{\sigma_{c a l}}{\sqrt{N_{c a l}}}
$$

which is approximately $0.8 \mathrm{MeV}$ for the peak+2 fills and $1.9 \mathrm{MeV}$ for the peak-2 fills, in the centre of mass energy $\left(\mathrm{E}_{\mathrm{CM}}\right)$. Secondly, the mean energy of the uncalibrated fills can also fluctuate with an error of

$$
\frac{\sigma_{\text {cal }}}{\sqrt{N_{\text {uncal }}}}
$$

When combined to form the error on the average energy of all fills, we expect fluctuations of

$$
\frac{N_{\text {uncal }}}{N_{\text {tot }}}\left(\frac{\sigma_{c a l}}{\sqrt{N_{c a l}}} \oplus \frac{\sigma_{\text {cal }}}{\sqrt{N_{\text {uncal }}}}\right)=\frac{\sigma_{\text {cal }} \sqrt{N_{\text {uncal }}}}{\sqrt{N_{\text {total }}} \sqrt{N_{c a l}}} .
$$

This implies errors of 0.7 and $1.6 \mathrm{MeV}$ in $\mathrm{E}_{\mathrm{CM}}$ for the peak+2 and peak-2 datasets, respectively. The peak point has a larger error of $5.4 \mathrm{MeV}$ because we have only one resonant depolarization calibration there. It is computed using the combined RMS scatter from all calibrations.

Using the combined RMS scatter for the off-peak points would give errors on the mean off-peak energies of $1.3 \mathrm{MeV}$, and would not change the final errors on $\mathrm{M}_{\mathrm{Z}}$ and $\Gamma_{\mathrm{Z}}$.

\subsection{Systematic effects due to imperfections in the model of energy variation}

There are a few ways in which we know the energy measurements are not exactly representative of physics conditions. Any systematic difference between calibration and physics conditions must be corrected for and can lead to a systematic error. The correction for any quantity, $\mathrm{X}$, whose variation can change the energy, is:

$$
\Delta E=\alpha_{X} \times\left(<X_{p h y s}>-<X_{c a l}>\right)
$$

where $\left\langle X_{\text {phys }}\right\rangle$ is the luminosity-weighted average of the quantity $X$ and $\left\langle X_{c a l}\right\rangle$ is the average of the same quantity at the time of the calibrations. Systematic errors arise from the limited knowledge of the coefficient $\alpha_{X}$. It is worth noticing that a wrong value of the coefficient $\alpha_{X}$ also increases the RMS scatter of the calibration data by a quantity $\Delta \alpha_{X} \times \sigma_{X}$ where $\sigma_{X}$ is the RMS of the quantity $\mathrm{X}$ at the time of the calibrations. This last contribution increases the systematic error assigned to the average energy of the fills resulting in a slight overestimate of this error.

In order to study quantitatively the systematic error due to the limited knowledge of parameters or to assumptions of the model, the complete chain of the program producing 


\begin{tabular}{|l|r|r|}
\hline strategy & peak-2 & peak+2 \\
\hline \hline no reference magnet temp. correction & +0.04 & -0.06 \\
\hline no dipole temperature correction & +0.61 & +1.47 \\
\hline no QFQD correction & +0.80 & +0.72 \\
\hline no tide correction & -0.40 & -4.10 \\
\hline no BOM correction & +0.34 & +1.01 \\
\hline Full NMR strategy & -1.07 & -0.84 \\
\hline NMR at start & +1.05 & +0.91 \\
\hline \hline
\end{tabular}

Table 3: Difference in MeV in the mean centre of mass energy of the two off-peak points when changing the model of the energy of LEP

the LEP energies has been rerun after introducing changes in the model. The differences with respect to our reference strategy are summarized in table 3 and are used in the following to assess some of the systematic errors.

The mean temperatures of the dipoles were slightly higher during the energy calibrations than during physics running. Since the correction factor is known to 25\% [7] this introduces a systematic error of $0.2 \mathrm{MeV}$ in $\mathrm{E}_{\mathrm{CM}}$ at peak-2 and $0.3 \mathrm{MeV}$ in $\mathrm{E}_{\mathrm{CM}}$ at peak +2 . The mean temperature difference is slightly different at each scan energy, but the uncertainty in the correction factor is correlated between the scan points. The scatter in the temperature measurements during calibrations is $0.17^{\circ} \mathrm{C}$, producing at most $1.7 \mathrm{MeV}$ in $\mathrm{E}_{\mathrm{CM}}$ contribution in quadrature to the energy scatter.

The calibration measurements do not sample in an unbiased way the variations of the current in the QFQD compensation coil, described earlier. As shown in table 3, not applying this correction results in variations of 0.8 and $0.7 \mathrm{MeV}$ in the mean centre of mass energies of the two off-peak points. The correction is known with an uncertainty of $50 \%$ (see subsection 4.1) implying a systematic error of $0.4 \mathrm{MeV}$ in $\mathrm{E}_{\mathrm{CM}}$, correlated between the two energies.

Although the physics fills average well over the typical 12 hour variation of the tide correction, this is not the case for the calibrations at peak +2 , resulting in a change of the mean energy by $-4 \mathrm{MeV}$ in $\mathrm{E}_{\mathrm{CM}}$ when the tide correction is neglected. This energy variation is compatible with the limited sampling of the calibrations. Since the tide coefficient has been measured to $10 \%$ [6], this results in a systematic error of $0.4 \mathrm{MeV}$ in $\mathrm{E}_{\mathrm{CM}}$ at peak +2 and less than $0.1 \mathrm{MeV}$ in $\mathrm{E}_{\mathrm{CM}}$ at peak-2.

The BOM measurements of the relative transverse orbit position have no identified additional systematic error because their scale factor is well understood both from theory and from measurement. Any fluctuation due to the finite reproducibility of the BOM system is automatically included in the scatter calculation.

As discussed previously, the observed average increase of the NMR measurement of the reference field is not understood. To cope with this uncertainty, the calculation of the energy includes one half of the rise observed in the NMR reading between the start of the fill and the current time. The effect on the mean energy of half of the observed mean rise of the NMR at each energy point is then taken as the systematic error from this correction. This mean rise is fully correlated between energies, and contributes a corresponding systematic error to the $\mathrm{M}_{\mathrm{Z}}$ measurement. The changes of the mean energy (0.9 $\mathrm{MeV}$ in $\mathrm{E}_{\mathrm{CM}}$ ) are similar between the peak+2 and peak-2 scan points, so this systematic error has little effect on the error on $\Gamma_{\mathrm{Z}}$. Since the mean NMR rise is taken 
from a distribution of finite variance, an error of $0.4 \mathrm{MeV}$ in $\mathrm{E}_{\mathrm{CM}}$, uncorrelated between scan points, is included which does affect the error on $\Gamma_{\mathrm{Z}}$. The correlated part of the error is then computed to be $0.8 \mathrm{MeV}$ in $\mathrm{E}_{\mathrm{CM}}$.

There are a number of parameters with no known effect on the beam energy, including time of day, day of week, total beam current and random orbit fluctuations. No significantly large variation of beam energy has been linked to any of these effects, but we proceed by attempting to estimate the size of the maximal variation permitted by the existing measurements. By fitting the observed energy data to each hypothetical source, we obtain an estimate of the size of the effect. This can then be used to set an upper limit on the effect of mis-sampling, taking into account the difference between the observed quantity in physics and in calibration. All the distributions we have examined are consistent with the energy measurements being an unbiased sample of the underlying distribution. We therefore assign no additional systematic error, relying on the scatter calculation to represent any effects that might be present.

\subsection{Systematic effects in the measurement of the energy}

Systematic differences between the conditions of LEP at the time of energy calibrations and those during physics running include changes to the tunes, the RF frequency and the orbit. Orbit distortions have to be introduced to compensate for the spin effects of the solenoids and to steer the beam into collision with the laser photons from the polarimeter. The individual studies of these differences are described in section 1 and in reference [2]. In most cases the only significant contribution to the systematic errors of the mean energy values arise from the errors common to all calibrations, since repetition reduces the effects of any random fluctuations of the measurements, such as those due to the finite bin size.

The errors due to the electron mass, revolution frequency, RF magnet frequency and the width of the excited resonance are treated as constant throughout the year, and correlated between energy points.

The effects due to quadratic nonlinearities and longitudinal fields can be calculated using only well known quantities and so the theoretical estimates of table 1 are used for these systematic errors.

Tune shifts produced by vertical orbit corrections produce a systematic error on the average of the calibrated fills of at most $1.6 \mathrm{MeV}$ in $\mathrm{E}_{\mathrm{CM}}$, which is reduced by the square root of the number of calibrated fills. They also contribute as much as $1.6 \mathrm{MeV}$ in $\mathrm{E}_{\mathrm{CM}}$ to the RMS scatter of the calibration measurements with respect to the model.

Combined, these errors correspond to a systematic error of approximately $0.5 \mathrm{MeV}$, uncorrelated between energies.

\subsection{Systematic errors on the positron beam energy}

Any unknown systematic energy difference between the electron and positron beams would create a systematic error, because the calibrations in 1993 were made using only the electron beam.

As already discussed in section 2, the energies of the two beams are considered to be fully correlated and a systematic error of $0.3 \mathrm{MeV}$ is assigned to their difference. As it is not known whether the peak +2 and peak -2 points have identical differences, a correlation of $50 \%$ is assigned between the systematic errors at the two energies. 


\subsection{Systematic errors due to RF effects}

The systematic errors on the energy corrections due to RF effects are the only errors which are not fully correlated amongst the four interaction points.

As described in section 3, the model used to describe the energy offset at the interaction points fits well with the measured quantities. The main source of error arises from uncertainties in the precise positions of the RF cavities. The total systematic error on the energy correction due to RF effects at the interaction points is $\pm 0.6 \mathrm{MeV}$ for L3 and OPAL and $\pm 1.0 \mathrm{MeV}$ for ALEPH and DELPHI. The energy variations due to RF effects are fully anti-correlated for ALEPH and DELPHI, but essentially uncorrelated for L3 and OPAL, since here the dominant error arises from uncertainties in the geometrical positions of individual cavities, and the other contributions to the error have correlated and anti-correlated parts of about equal size. These errors are essentially fully correlated between the energy scan points.

When the results of the four experiments are combined, the relevant values are the averages of the energies at the four interaction points. The systematic error due to RF effects on the average energies is reduced to $\pm 0.2 \mathrm{MeV}$, correlated between peak -2 and peak +2 .

\subsection{The covariance matrix}

The exact calculation of the errors on $\mathrm{M}_{\mathrm{Z}}$ and $\Gamma_{\mathrm{Z}}$ must be done from the covariance matrix, as the RF correction involves cancellations amongst the experiments. All other terms are identical from experiment to experiment. Table 4 is the covariance matrix to use when averaging the results of the four experiments. This matrix is calculated assuming approximately equal efficiencies for the four experiments, and that the events lost are approximately equally distributed across the year. As the error terms which vary from experiment to experiment are comparatively unimportant to the result, this is not a significant constraint. For example, the complete loss of the data from ALEPH or DELPHI would increase the systematic error on the $Z$ width by only $5 \%$ of its value.

\begin{tabular}{|l||r|r|r|}
\hline & peak-2 & peak & peak+2 \\
\hline \hline peak-2 & 4.00 & 1.02 & 1.01 \\
\hline peak & 1.02 & 29.18 & 1.09 \\
\hline peak+2 & 1.01 & 1.09 & 2.19 \\
\hline \hline
\end{tabular}

Table 4: Covariance matrix in $\mathrm{MeV}^{2}$ in $\mathrm{E}_{\mathrm{CM}}$ for the combined results from the four LEP experiments.

Applying this matrix to equations 1 and 2 results in systematic errors of approximately $1.5 \mathrm{MeV}$ on $\Gamma_{\mathrm{Z}}$ and $1.4 \mathrm{MeV}$ on $\mathrm{M}_{\mathrm{Z}}$.

In subsection 5.1 it has been shown that the RMS scatter of the polarization data measured at the peak -2 and at the peak +2 energy points are only barely compatible. The systematic errors have been evaluated assuming a different RMS scatter at the two energy points, and it has been stated in subsection 5.1 that using a common RMS scatter would lead to essentially the same final errors on $\mathrm{M}_{\mathrm{Z}}$ and $\Gamma_{\mathrm{Z}}$. However, the correlation coefficient, $\rho$, between the systematic error on the $Z$ mass and width would change noticeably. When evaluated using the individual scatters at the two energy points, $\rho=-0.27$. When evaluated using a single, combined scatter, $\rho=0.12$. This can be understood by noticing that an upward fluctuation in the peak +2 energy point increases both $\mathrm{M}_{\mathrm{Z}}$ and 
$\Gamma_{Z}$, while a similar fluctuation in the peak-2 energy point increases $M_{Z}$ while decreasing $\Gamma_{Z}$. If the scatter errors on the two energy points are equal, these effects cancel, and the only correlation is from other, smaller effects. If the scatter error at the peak +2 point is smaller than that at the peak -2 point, a negative correlation results. We conclude that the $\mathrm{M}_{\mathrm{Z}}-\Gamma_{\mathrm{Z}}$ correlation coefficient is uncertain at the level of \pm 0.3 .

The covariance matrix for any individual experiment can be computed to a good approximation from the matrix shown in table 4 by adding to each term the quadratic difference between the RF error relevant to that experiment (see table 2) and the RF error corresponding to the combined result (also shown in table 2). This quadratic difference amounts to $0.32 \mathrm{MeV}^{2}$ for L3 and OPAL and to $0.96 \mathrm{MeV}^{2}$ for ALEPH and DELPHI.

\section{Checks of the energy calibration}

This section describes a number of tests that have been performed to check the calibration procedure.

\subsection{Energy dependence of the measured cross sections}

Early in the analysis of the calibration data, a check was done to ensure that the cross sections measured by the experiments reflected the measured change of the LEP energy implied by the resonant depolarization calibrations. In order to avoid biasing later analyses, the four LEP experiments were asked to provide only the relative cross section ratios between a "low energy" sample of fills and a "high energy" sample for each of the two off-peak scan points.

Given the known $Z$ lineshape parameters, the combined cross section results implied changes in $\mathrm{E}_{\mathrm{CM}}$ of $27 \pm 7 \mathrm{MeV}$ and $21 \pm 7 \mathrm{MeV}$ at the peak -2 and peak +2 energy points, respectively. The calibration measurements predicted energy differences of $22 \pm 4$ and $18 \pm 4 \mathrm{MeV}$, respectively, in good agreement with the cross section data. The $\chi^{2}$ values for combining the data from the four experiments are 4.2 and 2.2 for 3 degrees of freedom, respectively.

\subsection{Flux loop calibrations}

The flux loop consists of closed electrical loops threading all the LEP dipoles and is used to measure the magnetic field of the bending magnets with a precision of about $\pm 10^{-4}$. More details on this instrument can be found in [7]. This method is insensitive to static magnetic fields and to the bending field components of quadrupoles and sextupoles on non-central orbits. Some corrections must be applied before it can be compared with the results from resonant depolarization calibrations. The measured flux loop energy, $E_{f l}$, must be corrected [7] by $7 \pm 8 \mathrm{MeV}$ to account for aging of the concrete-iron dipole magnet cores, for the Earth's magnetic field, for the effect of a nickel layer in the LEP vacuum chamber and for the difference between the operational RF frequency and the central frequency in the absence of tides. Figure 10 shows that flux loop and resonant depolarization calibrations are consistent. The RMS of the difference is $\sim 4.7 \mathrm{MeV}$ in beam energy, which corresponds to the precision of the flux loop calibration.

\subsection{Beam energy and tunes}

For a defined setting of the total bending field, energy variations, $\Delta E$, around the nominal energy, due to tides or orbit length variations, give rise to tune shifts

$$
\Delta Q=Q^{\prime} \frac{\Delta E}{E}
$$




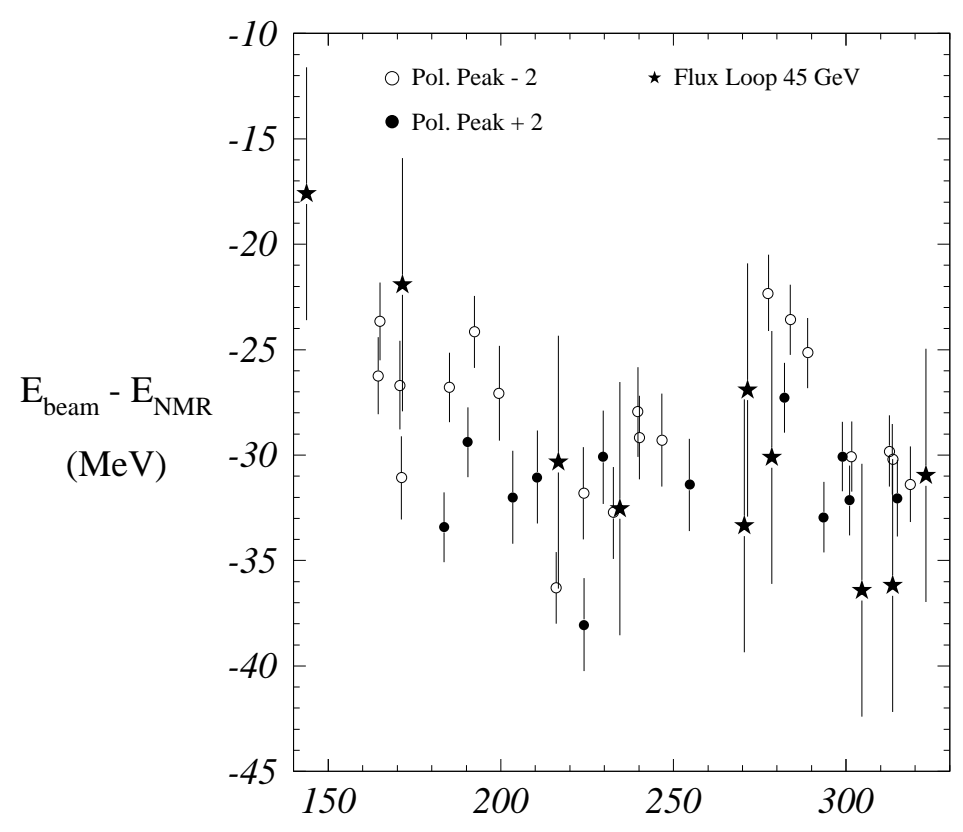

\section{Time (days)}

Figure 10: Flux loop magnet calibrations versus day in 1993 compared with beam energy measured with resonant depolarization and corrected for changes in the integrated dipole field and for orbit position in the quadrupoles. The scales in this figure are equivalent to those in figure 8.

where $Q^{\prime} \approx+2$ is the corrected chromaticity which includes quadrupole and sextupole effects. Such tune shifts are small since $\Delta E / E$ does not exceed $10^{-3}$. On the other hand, a change, $\Delta(B l)$, of the bending strength induces an energy shift $\Delta E / E=\Delta(B l) /(B l)$ and a tune shift

$$
\Delta Q=Q_{n}^{\prime} \frac{\Delta E}{E}
$$

where $Q_{n}^{\prime}$ is the natural quadrupole chromaticity: $Q_{n}^{\prime} \approx-120$ for both planes in LEP. In this case the strength of the quadrupoles is mismatched and the tune change is large and measurable. Since for every energy calibration the tunes were carefully set to $\left(Q_{x}, Q_{y}\right)=(90.1,76.2)$ by an adjustment of the current in the arc quadrupoles, a correlation is expected between the quadrupole current and $E$, provided that the changes in $E$ are caused by variations of the total field strength, $B l$.

For this analysis, the relative changes in quadrupole current settings of the focusing $(\mathrm{QF})$ and the defocusing (QD) quadrupoles have been averaged to improve the precision:

$$
\frac{\Delta I_{q}}{I_{q}}=\frac{1}{2}\left(\frac{\Delta I_{q f}}{I_{q f}}+\frac{\Delta I_{q d}}{I_{q d}}\right)
$$

The beam energies are corrected for tides, radial orbit shifts and $f_{R F}$, because these parameters affect the tunes through $Q^{\prime}$ and not $Q_{n}^{\prime}$. No correction is applied for LEP parameters that involve a change in the bending strength, since these should be seen by the quadrupole current. The slope of the correlation is expected to be

$$
\frac{\Delta E}{E} \approx 0.6 \frac{\Delta I_{q}}{I_{q}}
$$


The experimental slope is in agreement with the expectation and the correlation is good (figure 11). The precision is about $5 \times 10^{-5}$ and is limited by the accuracy of the tune setting.

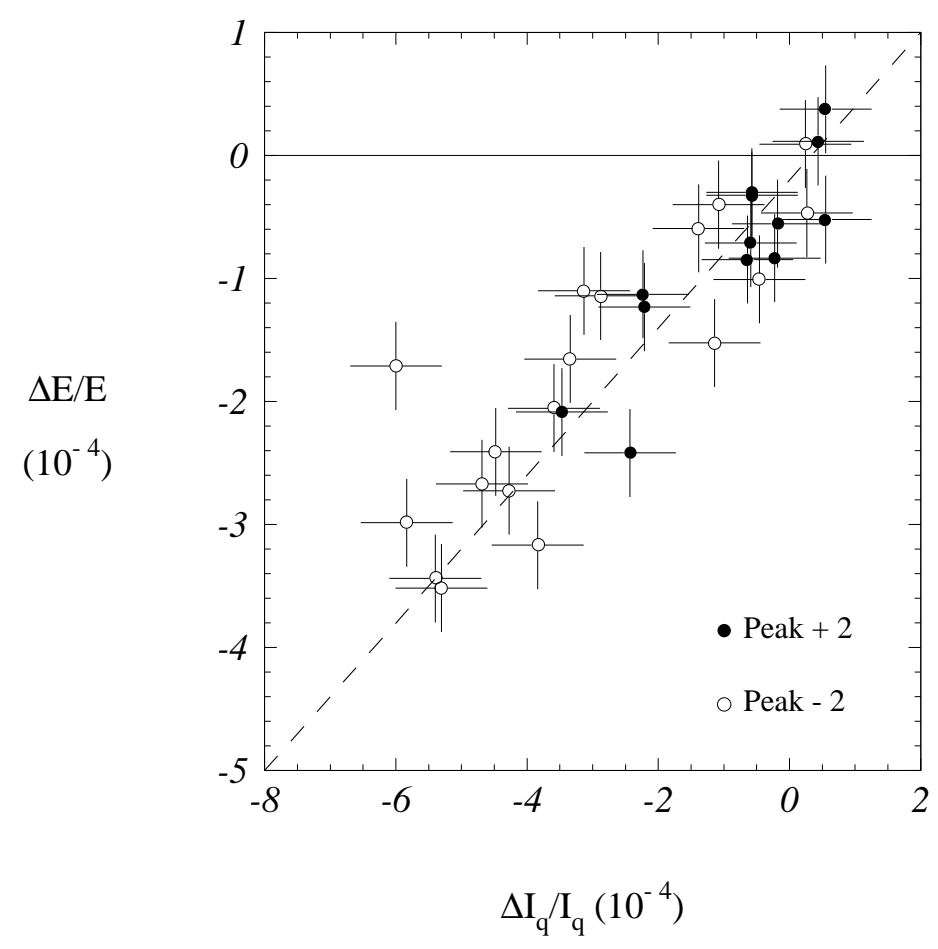

Figure 11: Comparison of quadrupole current and beam energy measured with resonant depolarization and corrected for position of the orbit inside the quadrupoles.

\subsection{Bunch length and energy spread}

The bunch length, $\sigma_{Z}$, can be directly related to the energy spread, $\sigma_{E}$, of the beams:

$$
\sigma_{E}=\frac{E}{\alpha_{c} R} Q_{s} \sigma_{Z},
$$

where $\alpha_{c}$ is the momentum compaction factor, $R$ is the bending radius of LEP and $Q_{s}$ is the synchrotron tune.

The bunch length can be observed most precisely from the RMS scatter of the positions of reconstructed Z decays in the experimental detectors. Studies done [22, 23] using the ALEPH and OPAL detectors have shown the expected effects on bunch length from changes in $Q_{s}$ and from the use of wigglers early in the fill. For several fills, these studies have been confirmed by a direct measurement of the bunch length using a streak camera [24]. From these measurements, the average value of the spread in the centre of mass energy is found to be $55 \pm 5 \mathrm{MeV}$.

In addition, $\sigma_{E}$ is sensitive to variations in the energy of the beams due to changes in the ring circumference. When the beam is offset from the centre of the quadrupole magnets the damping partition number changes. A $10^{-4}$ change in beam energy corresponds to a $1 \%$ change in bunch length. The effect of earth tides on the bunch length is visible in the data, as is the variation recorded by the BOM system over the course of the year. 


\section{$7 \quad$ Calibration of the peak energy points}

A centre of mass energy calibration of about $\pm 20 \mathrm{MeV}$ precision is required at the maximum of the cross section in order to avoid introducing significant systematic errors into the measurements of the forward-backward charge asymmetries and the absolute cross section at the $\mathrm{Z}$ peak.

The energies in peak physics fills during the scan were calculated using the same model of energy variation as was used for the off-peak fills (see section 4). The systematic error on the average peak energy (see table 4 ) is three times larger than those on the off-peak points since only one energy calibration was performed at peak energy and the conditions of LEP were less stable.

About half of the 1993 statistics at peak energy was collected before the scan started, when the logging system of LEP was not yet fully commissioned. It is therefore not feasible to use the same detailed model of energy variation for the pre-scan dataset. A much simpler analysis, similar to that used for the 1992 LEP energy calibration [8], has been made instead. There were six resonant depolarization energy calibrations made during the pre-scan period. These were all done during machine-development fills, not under physics running conditions and at the peak -2 energy point. Combining these, the mean difference between the calibrated beam energy and that measured by the flip-coil fielddisplay system [8] at the start of each fill was found to be $E_{\text {pol }}-E_{F D}=-28.4 \mathrm{MeV}$, with an RMS scatter of $3.0 \mathrm{MeV}$. Taking into account the nonlinearity of $E_{\text {pol }}-E_{F D}$, as determined from the measured energy offset between the peak and peak-2 points, it was found that the pre-scan energy calibration was consistent with that of the 1992 LEP data [8]. This same calibration was therefore kept for the 1993 pre-scan dataset: $\mathrm{E}_{\mathrm{CM}}=2 E_{F D}-67 \mathrm{MeV} \pm 18 \mathrm{MeV}$, where $\mathrm{E}_{\mathrm{CM}}$ is the corrected centre of mass energy for a fill and $E_{F D}$ is the field-display beam energy measured at the start of the fill. The interaction point dependent RF correction (+20 MeV for L3 and OPAL) must also be added to the above correction.

The quoted uncertainty of $\pm 18 \mathrm{MeV}$ is very conservative for the 1993 pre-scan, but it is already small enough that it contributes an insignificant error to the electroweak parameters determined from the combined LEP data.

\section{Comparison with previous calibrations}

Figure 12 compares the LEP energy calibrations from 1991 through to the present. Although the method of calculating the energy has become more sophisticated with time, the overall agreement is satisfying. In particular, including the effects of ground motion via the tide and BOM corrections has not resulted in large shifts in the average energy.

The 1991 calibration resulted in errors on $\Gamma_{\mathrm{Z}}$ that were dominated by magnetic uncertainties, in particular the magnetic local energy scale when extrapolating from the peak +2 energy where the depolarization calibrations were done. This nonlinearity resulted in a shift of the peak-2 energy by $9 \pm 5 \mathrm{MeV}$ in $\mathrm{E}_{\mathrm{CM}}$ with respect to the reading of the reference magnet (flip-coil). This is consistent with the 1993 calibration value of $5.8 \pm 2.0 \mathrm{MeV}$. The 1991 calibration resulted in an error on $\mathrm{M}_{\mathrm{Z}}$ dominated by the scatter of the calibrations $(3.7 \mathrm{MeV})$, by temperature corrections $(3 \mathrm{MeV})$ and by the magnetic local energy scale $(3 \mathrm{MeV})$. These calibrations were not corrected for tidal variations. The systematic errors on the energy therefore have a negligible correlation between the 1991 and 1993 scans.

The 1992 calibration is dominated by the error due to the scatter of the depolarization measurements. It is possible that this is due to ground motions of the type seen in 


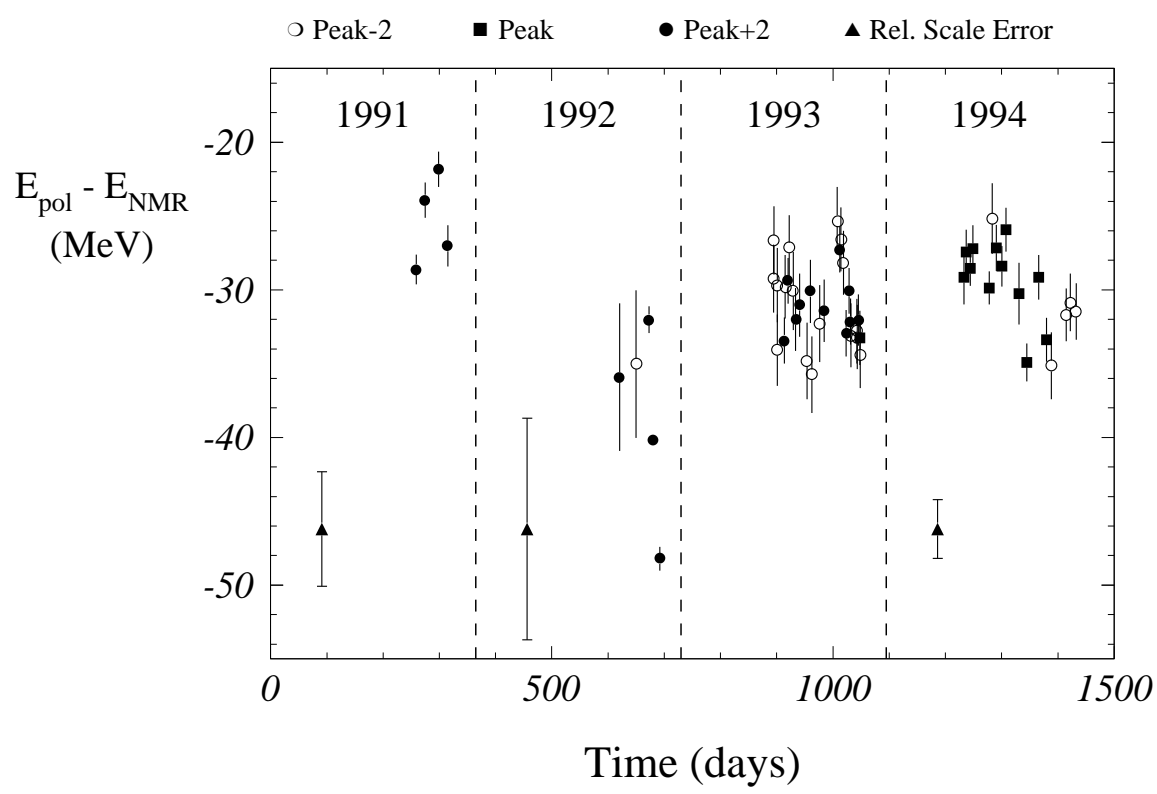

Figure 12: Comparison of LEP energy calibrations versus time. On the vertical axis is plotted the difference between the calibrated beam energy and the value observed in the reference magnet. Each point corresponds to a fill with energy measured by resonant depolarization. Only corrections used in the calibration of the data for each year have been applied to the points. For example, the 1991 points do not include tide corrections. The error bars near the 1991, 1992 and 1994 points (relative scale error) represent the systematic errors when comparing all the points in those years to the data of 1993.

1993. Unfortunately, the BOM system during 1992 and earlier has drifts and discontinuities in its calibration data which makes it unusable for precise measurements over long periods. There is also a significant error due to the magnetic uncertainties, in particular the nonlinearity error which also appears in the 1991 calibration. These errors are completely uncorrelated with the procedure and fluctuations in 1993, so again the correlation between the 1992 and 1993 energy errors is negligible.

The depolarization procedure now includes additional cross-checks. The records of the 1991 and 1992 depolarization measurements have been re-examined and there is no reason to believe that there are any problems with these measurements.

It would be difficult at this point to recalculate the energies of previous data using the current methods, as much of the information now used was not acquired at the time. In particular, the BOM system upgrade and the improvements in the logging system were vital to the success of the 1993 calibration method, and are unavailable for prior years.

To summarize, the 1991 and 1992 energy calibrations are still believed to be correct within their stated errors. They have negligible correlation with the 1993 scan energy errors.

A global fit to the data collected by the four experiments in 1992 and 1993 and using 1992 and 1993 calibrations results in systematic errors of $1.4 \mathrm{MeV}$ on $\mathrm{M}_{\mathrm{Z}}$ and $1.6 \mathrm{MeV}$ on $\Gamma_{Z}$, in agreement with the values computed in section 5.6 using equations 1 and 2 . The data collected in 1992 and 1993 pre-scan periods were taken at an energy very close to the maximum cross section. At this energy point the contribution of the corresponding error on energy to the errors on the $\mathrm{Z}$ mass and width is small. However, in order to ensure energy calibration by resonant depolarization, the peak point data during the scan were 
taken at an energy about $100 \mathrm{MeV}$ below the maximum cross section. The fit results for the $\mathrm{Z}$ width are more sensitive to the energy error on this point. When only 1993 scan data are used in the fit the error on $\mathrm{M}_{\mathrm{Z}}$ is unchanged but the systematic error on $\Gamma_{Z}$ increases to $1.9 \mathrm{MeV}$.

\section{Conclusions}

The procedure to calibrate the energy of the LEP beams adopted during the 1993 energy scan resulted in a significant improvement in precision with respect to the one used during the 1991 energy scan.

The resulting systematic error on the width of the $Z$ boson is substantially smaller than the statistical error. The systematic error on the mass of the $\mathrm{Z}$ boson has been reduced by a factor of five compared to the 1991 scan and it is now also smaller than the statistical error.

The main source of systematic error is linked to the determination of the mean energy of the uncalibrated fills, which is estimated from the calibrated ones. This error depends on the statistics of the calibrated fills and on the RMS scatter of the deviations of the calibrations with respect to the model of the energy variation with time.

Adopting the same calibration procedure in a possible future energy scan would result in a small correlation with the 1993 scan allowing a further important reduction of the overall errors on $\mathrm{M}_{\mathrm{Z}}$ and $\Gamma_{\mathrm{Z}}$.

\section{References}

[1] L. Arnaudon et al: "Measurement of LEP beam energy by resonant spin depolarization". Phys. Lett. B 284 (1992) 431-439.

[2] L. Arnaudon et al.: "Accurate Determination of the LEP Beam Energy by Resonant Depolarization". CERN SL/94-71 (BI). To be published in Zeitschrift für Physik C.

[3] R.Billen et al: "LEP accelerator logging system using on-line database" International Conference on Accelerator and Large Experimental Physics Control Systems(ICALEPC 93)- Berlin- October 18-22 1993.

[4] L. Arnaudon et al: "Measurement of the mass of the $\mathrm{Z}$ boson and the energy calibration of LEP" Phys. Lett. B 307 (1993) 187-193.

[5] M. Koratzinos: "Electroweak results from LEP", presented at the La Thuile Conference, 1994

[6] L. Arnaudon et al: "Effects of Terrestrial Tides on the LEP Beam Energy". CERN SL/94-07 (BI). To be published in Nuclear Instruments and Methods.

[7] L. Arnaudon et al (The working group on LEP Energy): "The Energy Calibration of LEP in 1991", CERN-PPE/92-125, CERN-SL/92-37 (DI), contributed to the XXVIth International Conference on High Energy Physics, Dallas, July 1992.

[8] L. Arnaudon et al (The working group on LEP Energy) "The Energy calibration of LEP in 1992". CERN-SL/93-21 (DI).

[9] A.A. Sokolov and I.M. Ternov: "On Polarization and Spin Effects in the Theory of Synchrotron Radiation". Sov. Phys. Dokl. 8(1964)1203.

[10] The Particle Data Group, Phys. Rev. D50 (1994).

[11] R. Assmann et al.: "Polarization Studies at LEP in 1993". CERN SL/94-08 (AP).

[12] G. Alexander et al: "Measurements of Polarization in LEP". In "Polarization at LEP", G. Alexander et al, eds., CERN Yellow report 88-06 (1988) Vol.II,3. 
[13] J. Buon: "Interference Effects between Depolarization Resonances and Higher-Order Corrections to Perturbed Spin Motion in Synchrotons and Storage Rings". LALRT 87-09.

[14] I.A. Koop et al.: "Investigation of the Spin Precession Tune Spread in the Storage Ring". Proc. $8^{\text {th }}$ International Symposium on High Energy Spin Physics, Minneapolis, September 12-17, 1988. AIP Conf. Proc. No. 187, p. 1023.

[15] J.P. Koutchouk: "Spin Tune Shifts due to Solenoids". CERN-SL/93-26 (AP), 1993.

[16] R. Assmann and J.P. Koutchouk: "Spin Tune Shifts Due To Optics Imperfections". CERN SL/94-13 (AP).

[17] K.A.Drees: "Energy Difference of Electron and Positron Beam", CERN SL/Note 94-100 (BI).

[18] M. Placidi et al: "Polarization Results and Future Perspectives", Proc. of the Third Workshop on LEP performance, ed. J. Poole, CERN SL/93-19.

[19] P. Melchior: "Tidal Interactions in the Earth Moon System", Communications, Serie B, No 160, IUG General Assembly, Vienna, August 1991. Fortran code courtesy of P. Melchior, Observatoire Royal de Belgique.

[20] R. Jacobsen: "Observations of Tidal Distortions of the LEP Orbit". CERN-SL-MD Note 62, May 1993.

[21] J. Wenninger: "Study of the LEP Beam Energy with Beam Orbits and Tunes". CERN-SL/94-14 (BI), 1994.

[22] R. Jacobsen: "1993 LEP Energy Spread and Variation From Spot Size Measurements", ALEPH 94-060, April 1994.

[23] D. Strom: "Determination of the LEP energy spread from the longitudinal beam size in OPAL", Opal Technical Note TN264, November 1994.

[24] E. Rossa: "Real Time Single Shot Three-Dimensional Measurement of Picosecond Photon Bunches", CERN SL/94-79 (BI).

K. Hanke: "Measurement of the bunch length at LEP with a streak camera and comparison with results from LEP experiments", Diploma work, PITHA 94/1, May 1994, RWTH Aachen, Germany. 